\title{
Bifurcation phenomena in viscoelastic flows through a symmetric 1:4 expansion
}

\author{
Gerardo N. Rocha ${ }^{\mathrm{a}}$, Robert J. Poole ${ }^{\mathrm{b}}$, Paulo J. Oliveira ${ }^{\mathrm{a}, *}$ \\ ${ }^{a}$ Unidade Materiais Têxteis e Papeleiros, Departamento de Engenharia Electromecânica, Universidade da Beira Interior, 6201-001 Covilhã, Portugal \\ ${ }^{\mathrm{b}}$ Department of Engineering, Mechanical Engineering, University of Liverpool, Brownlow Hill, Liverpool L69 3GH, UK
}

Received 12 May 2006; received in revised form 26 July 2006; accepted 22 August 2006

\begin{abstract}
In this work we present an investigation of viscoelastic flow in a planar sudden expansion with expansion ratio $D / d=4$. We apply the modified FENE-CR constitutive model based on the non-linear finite extensibility dumbbells (FENE) model. The governing equations were solved using a finite volume method with the high-resolution CUBISTA scheme utilised for the discretisation of the convective terms in the stress and momentum equations. Our interest here is to investigate two-dimensional steady-state solutions where, above a critical Reynolds number, stable asymmetric flow states are known to occur. We report a systematic parametric investigation, clarifying the roles of Reynolds number $(0.01<R e<100)$, Weissenberg number $(0<W e<100)$ and the solvent viscosity ratio $(0.3<\beta<1)$. For most simulations the extensibility parameter of the FENE model was kept constant, at a value $L^{2}=100$, but some exploration of its effect in the range 100-500 shows a rather minor influence. The results given comprise flow patterns, streamlines and vortex sizes and intensities, and pressure and velocity distributions along the centreline (i.e. $y=0$ ). For the Newtonian case, in agreement with previous studies, a bifurcation to asymmetric flow was observed for Reynolds numbers greater than about 36 . In contrast viscoelasticity was found to stabilise the flow; setting $\beta=0.5$ and $W e=2$ as typical values, resulted in symmetric flow up to a Reynolds number of about 46 . We analyse these two cases in particular detail.
\end{abstract}

(C) 2006 Elsevier B.V. All rights reserved.

Keywords: Planar symmetric expansion; Asymmetric flow; Viscoelastic fluid; FENE-CR model; FENE-MCR model; Finite volume method

\section{Introduction}

Numerical simulation of viscoelastic flows has been used increasingly for the analysis and understanding of fluid behaviour in a variety of processes of both industrial and scientific interest. From a fundamental point of view, viscoelastic fluid flow through ducts with abrupt change of cross-section, either expansions or contractions, are important as they highlight many of the unusual phenomena brought about by elasticity. These phenomena include complex recirculation patterns, not found with Newtonian fluids, vortex enhancement or suppression, the possibility of unsteady flow due to elastic instabilities, complex stress behaviour near geometrical singular points, etc. In addition, expansion and contraction geometries are relevant in engineering applications, particularly in the process industries, for example the channel feeding an extrusion die is unavoidably

\footnotetext{
* Corresponding author. Tel.: +351 275329 946; fax: +351 275329972.

E-mail addresses: gerardorocha@ portugalmail.pt (G.N. Rocha), robpoole@liv.ac.uk (R.J. Poole), pjpo@ubi.pt (P.J. Oliveira).
}

endowed with such localised perturbations in cross-section in order to achieve the desired extruded shape. A survey of the specialised literature shows that contraction flows of viscoelastic liquids have received a great deal of attention during the past 10-15 years, but studies (both numerical and experimental) of expansion flows are rather scarce. Given the rich fluid dynamic behaviour that has been observed in viscoelastic fluid flow in contractions, referred to above, this is perhaps not surprising (see the many examples in the book of Boger and Walters [1] for example).

Of the few papers that have investigated viscoelastic fluid flow through expansions they are, in the main, concerned with creeping flow conditions (i.e. vanishing $R e$ ). The numerical works of Darwish et al. [4] and Missirlis et al. [5] both use a finite volume technique to simulate viscoelastic fluid flow through a two-dimensional 1:4 plane sudden expansion using the UCM model for $R e=0.1$. Missirlis et al. [5] show that vortex activity is suppressed with increasing Deborah number (defined by the ratio between the characteristic time of the deformation process being observed and the characteristic time of the material) and that, as the Deborah number is increased beyond a critical 
value of 3.0, the recirculation zone is completely eliminated. The related works of Townsend and Walters [6] and Baloch et al. [7] are also worthy of mention. Both works used the linear form of the PTT model in an attempt to simulate the flow visualisations (shown originally in Townsend and Walters [6]) for the flow of two polymer solutions (polyacrylamide and xanthan gum) through both quasi two- and three-dimensional expansions. The visualisations clearly show the reduction in recirculation for the viscoelastic fluids, and the simulations are in good qualitative agreement with these visualisations.

For Newtonian fluids, as is well-known (first documented in Abbott and Kline [8]), above a critical Reynolds number the flow field downstream of the expansion exhibits a stable asymmetric flow state. The critical Reynolds number at which the flow becomes asymmetric is dependent on the expansion ratio (i.e. the ratio of the downstream to upstream channel heights) and, for three-dimensional flows, the aspect ratio (i.e. the ratio of channel width to inlet channel or step height). Indeed the asymmetry is completely absent for expansion ratios less than 1.5 . This asymmetry has been observed in both experimental (Cherdron et al. [9], Durst et al. [10] for example) and numerical (Drikakis [2], Battaglia et al. [3]) investigations. Drikakis conducted an extensive study on the effect of expansion ratio and was able to demonstrate that the critical Reynolds number for asymmetric flow to occur decreases with increasing Reynolds number. For the 1:4 expansion he obtained a critical $R e$ of 35.3. For the same expansion ratio Battaglia et al. obtained a slightly higher value of 35.8. In the current study, as we discuss in detail in Section 5.1 , we obtained a critical Reynolds number $R e_{\mathrm{cr}}=36$, in very close agreement with these studies.

Oliveira [11] was the first author to investigate viscoelastic fluid flow at high enough Reynolds numbers for asymmetric flow to be observed. Using the modified FENE-CR model the behaviour of viscoelastic fluids in a 1:3 planar sudden expansion was studied. At low Reynolds number, Oliveira was able to confirm the results of previous studies: namely the effect of elasticity is to reduce both the degree and magnitude of recirculating fluid downstream of the expansion compared with the Newtonian case. At high Reynolds numbers, although the asymmetry still occurred, the effect of elasticity was seen to be a stabil- ising one, i.e. the bifurcation to asymmetry flow occurred at higher Reynolds numbers for the viscoelastic cases. The critical Reynolds number was seen to be dependent on the Weissenberg number and the $\beta$ and $L^{2}$ parameters of the FENE-CR model.

In the current study we present a systematic numerical investigation of the flow of a FENE-MCR liquid in a planar sudden expansion of expansion ratio 4 . The basic elements of laminar flow, with moderate inertia, $\operatorname{Re} O(50)$ say, through a planar sudden expansion are illustrated in Fig. 1. The flow progresses from being fully developed at a plane some distance $L_{1}$ upstream from the expansion to being fully developed in the downstream channel at a distance $L_{2}$ from the expansion plane. The exact shape of the recirculation regions may be concave or convex with respect to the expansion corner, depending if the flow is dominated by viscous or inertial forces, respectively. The purpose of the present work is to provide quantitative data of benchmark quality for the flow through a 1:4 planar expansion of viscoelastic liquids obeying the constant viscosity FENE-MCR constitutive model.

The main objectives of the present study are: (i) to examine the possible effects of each non-dimensional parameter (i.e. $R e, W e$ and $\beta$ ) of the FENE-MCR model upon the flow and compare with the results of Oliveira [11] for a planar sudden expansion of lower expansion ratio of 1:3; (ii) to investigate the critical Reynolds number of the symmetry-breaking bifurcation and flow asymmetries occurring in plane sudden expansions for Newtonian and viscoelastic fluids; (iii) to analyse the effect of elasticity on the flow field; (iv) to show the variation of profiles of the velocity, stress and pressure along the centreline for the Newtonian and viscoelastic cases.

\section{Conservation and constitutive equations}

In the present work we consider the two-dimensional isothermal flow of an incompressible liquid flowing from a straight channel of height $d$ to a larger channel of height $D=4 d$, corresponding to an expansion ratio $D / d=4$. In consequence, the process generates a complex flow exhibiting regions of strong shearing near the walls and uniaxial planar extension along the centreline. The upstream channel where the cross-section

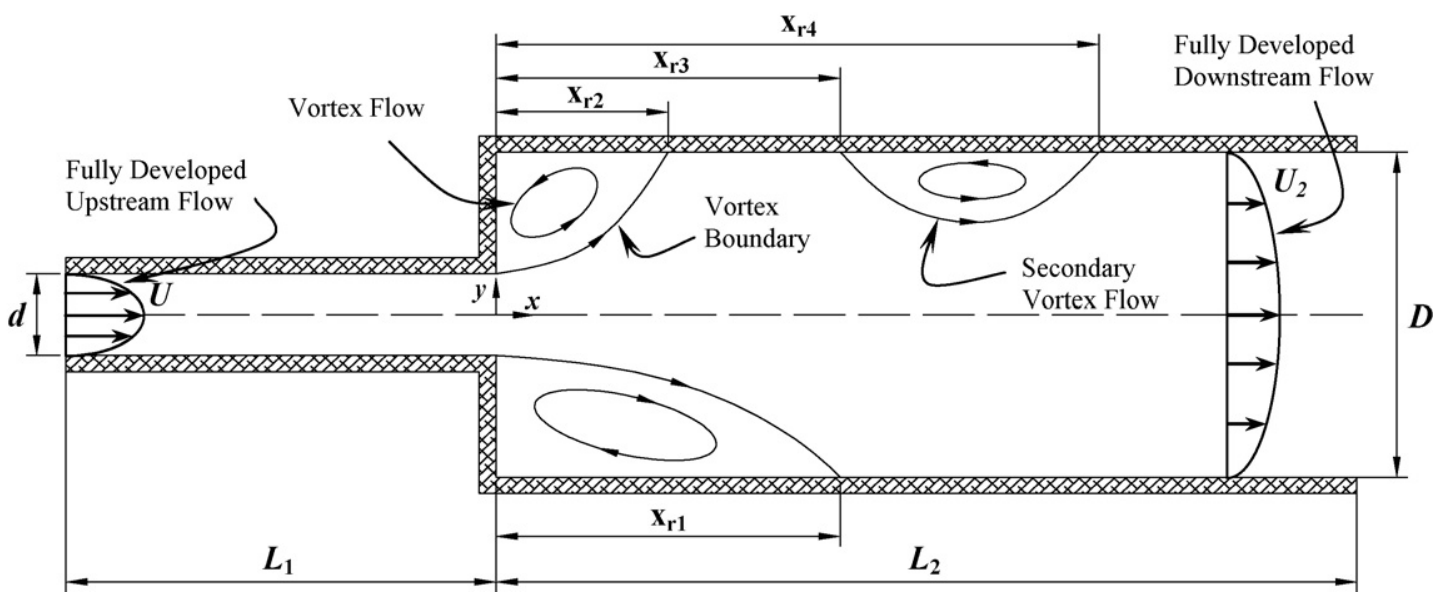

Fig. 1. Sudden expansion flow geometry including schematic of expected flow patterns for moderate $\operatorname{Re}(O(50))$. 
average velocity is $U$, has a length of $L_{1}=20 d$ and the downstream channel a length $L_{2}=50 d$, as show in Fig. 1. These lengths are required for the establishment of a clear region of fully developed flow upstream of the expansion, and complete flow redevelopment downstream of it.

This problem is governed by the usual equations of continuity and motion, which can be written as [12]:

$\nabla \cdot \boldsymbol{u}=0$

$\rho \frac{\mathrm{D} \boldsymbol{u}}{\mathrm{D} t}=-\nabla p+\nabla \cdot \boldsymbol{\tau}_{\mathrm{tot}}$

where $\boldsymbol{u}$ is the local velocity vector, $\rho$ the fluid density (assumed constant), $p$ the pressure, $\boldsymbol{\tau}_{\text {tot }}$ the total extra stress tensor, and $\mathrm{D}() / \mathrm{D} t=\partial() / \partial t+\boldsymbol{u} \cdot \nabla()$ is the substantial derivative, or derivative following the motion. For a homogeneous polymeric solution the extra stress can be decomposed by the sum of a Newtonian solvent and a polymeric solute contribution $\left(\boldsymbol{\tau}_{\text {tot }}=\boldsymbol{\tau}_{\mathrm{s}}+\boldsymbol{\tau}\right)$.

The Newtonian solvent component is expressed in Eq. (3), where the solvent viscosity $\eta_{\mathrm{s}}$ is constant, $\nabla \boldsymbol{u}^{\mathrm{T}}$ the transpose of the velocity gradient, and $\boldsymbol{D}$ is the rate-of-strain tensor.

$\boldsymbol{\tau}_{\mathrm{s}}=\eta_{\mathrm{s}}\left(\nabla \boldsymbol{u}+\nabla \boldsymbol{u}^{\mathrm{T}}\right) \equiv 2 \eta_{\mathrm{s}} \boldsymbol{D}$

When the fluid is viscoelastic (i.e. it presents simultaneously viscous and elastic properties), the problem is considerably more complicated compared to the Newtonian case.

In the current study we use a modified form of the finite extensibility non-linear dumbbells (FENE [13]) model, valid for polymeric materials, the so-called FENE-CR model, proposed by Chilcott and Rallison [14]. The FENE-CR model predicts constant shear viscosity, $\eta_{0}=\eta_{\mathrm{p}}+\eta_{\mathrm{s}}$, shear-thinning of the first normal-stress difference coefficient and bounded elongational viscosity (proportional to $L^{2}$ ) and is given by:

$\boldsymbol{\tau}+\frac{\lambda}{f(\tau)} \stackrel{\nabla}{\boldsymbol{\tau}}=2 \eta_{\mathrm{p}} \boldsymbol{D}$

with the stretch function $f(\tau)$ expressed by:

$f(\tau)=\frac{L^{2}+\left(\lambda / \eta_{\mathrm{p}}\right) \operatorname{tr}(\boldsymbol{\tau})}{L^{2}-3}$

In the previous equations, $\operatorname{tr}$ is the trace operator, $\lambda$ a constant relaxation time, $\eta_{\mathrm{p}}$ the polymer viscosity (constant) and $L^{2}$ is the extensibility parameter that measures the size of the polymer molecule in relation to its equilibrium size. The symbol ' $\nabla$ ' in Eq. (4) is used to denote Oldroyd's upper convected derivate:

$\underset{\boldsymbol{\tau}}{\nabla}=\frac{\mathrm{D} \boldsymbol{\tau}}{\mathrm{D} t}-\boldsymbol{\tau} \cdot \nabla \boldsymbol{u}-\nabla \boldsymbol{u}^{\mathrm{T}} \cdot \boldsymbol{\tau}$

and the superscript ' $\mathrm{T}$ ' in Eq. (6) denotes the transpose of a tensor. In the current study an additional simplification is embodied in Eq. (4), compared with the original FENE-CR equation of Chilcott and Rallison [14], in that the term $\mathrm{D}(1 / f) / \mathrm{D} t$ is considered to be negligible. This model is denoted FENE-MCR, for modified Chilcott-Rallison model. The FENE-CR and FENE-MCR models are identical in simple steady-state flows and the only difference between the two models occurs in complex transient flows, where the effect of the neglected term $(\boldsymbol{u} \cdot \nabla(1 / f))$ can be important only in strong convective flows. The FENE-MCR model has been used in previous works by Coates et al. [15] in a numerical study of axisymmetric contraction flow and more recently by the related study of Oliveira [11]. In other works Oliveira has shown that results from those two models are virtually undistinguishable in complex flows at low Reynolds numbers and since we wanted to compare the present simulations with those of [11] we decided to use the MCR model. However, some calculations were undertaken with the unmodified CR model (Eq. (4), with the $f(\tau)$ function inside the Oldroyd derivative) which lead essentially to the same results, with only very minor quantitative deviations with increasing $R e$.

The relevant non-dimensional parameters to be varied in this work are:

- $L^{2}$, the extensibility parameter of the FENE-CR model (base value fixed at $L^{2}=100$ );

- $\beta=\eta_{\mathrm{s}} / \eta_{0}$, the solvent viscosity ratio, where the global shear viscosity is $\eta_{0}=\eta_{\mathrm{s}}+\eta_{\mathrm{p}}$ (constant);

- $R e=\rho U d / \eta_{0}$, the Reynolds number;

- $W e=\lambda U / d$, the Weissenberg number.

\section{Numerical method}

As mentioned previously, the numerical method applied in this work is the finite volume method (FVM). The governing equations (Eqs. (1), (2), (4) and (5)) are discretised in space by integration over the set of control volumes forming the computational mesh, and in time over a small time step, $\Delta t$. This process results in systems of linearised algebraic equations for the equations of mass and momentum conservation jointly with the constitutive equation. In these equations all variables are evaluated and stored in the central position of the control volumes (cells) and the computational mesh applied for the present simulations is orthogonal. As a consequence, special procedures are required to ensure the pressure/velocity coupling and the velocity/stress coupling (following the Oliveira et al. method [16]).

For the calculation of the convective terms in both the constitutive Eq. (4) and the momentum Eq. (2) we use a high-resolution scheme called CUBISTA [17], with third-order accuracy in space for smooth flow, and having simultaneously both high numerical precision and good characteristics of iterative convergence. The CUBISTA scheme is implemented explicitly, except for the part corresponding to upwind fluxes which are incorporated implicitly through the coefficients.

The constitutive and the momentum conservation equations in discretised form are solved using a modified algorithm based on the SIMPLE algorithm developed by Patankar and Spalding [18] that allows, through an iterative process of pressure correction, to guarantee the coupling of velocity and pressure, verifying the continuity equation. The presence of the constitutive equation for the viscoelastic fluid requires some minor alterations to the original SIMPLE method, which are mainly concerned with the calculation of pressure from the continuity equation. Two new steps are introduced in the initial part of the algorithm to account for the stress equation; this procedure is documented in detail elsewhere [16]. 
Boundary conditions are required around the flow domain represented in Fig. 1. At the inlet of the channel, $x / d=-20$, we impose fully developed profiles for all non-zero variables $\left(u, \tau_{x x}\right.$ and $\left.\tau_{x y}\right)$. The relevant equations are given in Oliveira [11] and are not repeated here for conciseness. At $x / d=+50$, in the outlet channel, we impose the well-known boundary condition of vanishing axial variation for all quantities, i.e. $\partial / \partial x=0$, except the pressure which was linearly extrapolated from the inside channel. We confirmed that this outlet condition did not affect the main flow characteristics near the expansion, once $L_{2}$ is sufficiently long. Finally at the solid walls we impose the no-slip boundary condition.

\section{Computational meshes and accuracy}

In this section we provide some details about the computational meshes used in this work and, based on the results obtained for each mesh, we quantify the numerical accuracy. As we are primarily interested in bifurcations to asymmetric flow, the whole flow domain was simulated, i.e. we did not assume symmetry about the centreline (i.e. $y=0$ ). The computational mesh is comprised of four blocks, presented in Fig. 2, and their geometric characteristics are provided in Table 1.

Three computational meshes have been employed in this study and their main characteristics are given in Table 1. The table includes the number of cells for each block, $N_{x}$ along the $x$-direction, $N_{y}$ along the $y$-direction and the total number of cells or control volumes (NC) inside the computational domain. The number of degrees-of-freedom (DOF), for each mesh, is obtained through the multiplication of $\mathrm{NC}$ for the six variables (two velocity components, pressure and three stress tensor components) which compose the two-dimensional geometry.

The minimum cell size $\left(\Delta x_{\min }=\Delta y_{\min }\right.$, these values are normalised with $d$ ) near to the expansion is given in Table 1 , as are the expansion or compression factor $\left(f_{x}=\Delta x_{i} / \Delta x_{i-1}\right)$ for the cell size along the streamwise $x$-direction (i.e. the mesh is nonuniform). Along the $y$-direction we applied a uniform mesh and the expansion or compression factor $\left(f_{y}\right)$ is equal to 1, see Fig. 3 .

All the results to be presented in this study were calculated using the medium mesh (Mesh 2), and the fine (Mesh 3) and coarse (Mesh 1) meshes were obtained by doubling or halving, respectively, the number of cells along the $x$ - and $y$-direction, so as to enable quantification of numerical accuracy.

A schematic representation of the computational mesh (medium mesh-Mesh 2) used in the computational calculations of the main variables is presented in Fig. 3. This figure illustrates the local refinement of the mesh near the expansion plane $(x=0)$, where the highest stress gradients are expected to occur due to the abrupt increase in channel height.

The results obtained for the three computational meshes are presented in terms of vortex size and intensity in Table 2, for the Newtonian and a representative viscoelastic fluid $(R e=20$, $L^{2}=100, \beta=0.5$ and $\left.W e=2\right)$. With each refinement the number of cells in each direction is doubled and the geometric factor $\left(f_{x}\right.$, expansion or compression of cells) is the square root of the value used in the previous mesh. This procedure is useful for applying Richardson's extrapolation [19] technique for the convergenceorder accuracy in the numerical approximation. By assuming second-order accuracy, based on previous works with the same code $[11,20,21]$, the extrapolated values denoted by "Richard-

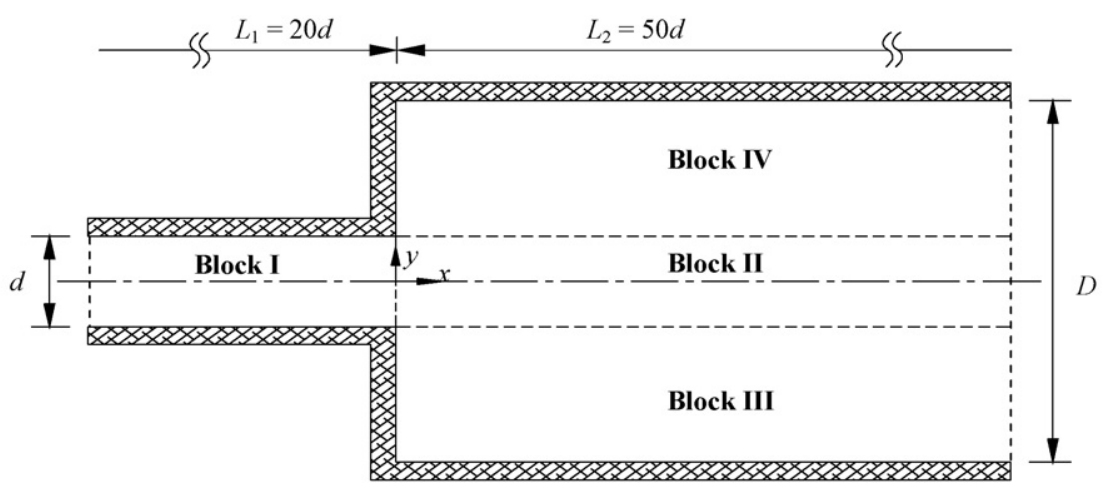

Fig. 2. Schematic representation of blocks in the 1:4 planar expansion.

Table 1

Main characteristics of computational mesh

\begin{tabular}{|c|c|c|c|c|c|c|}
\hline & \multicolumn{2}{|l|}{ Mesh 1} & \multicolumn{2}{|l|}{ Mesh 2} & \multicolumn{2}{|l|}{ Mesh 3} \\
\hline & $N_{x} \times N_{y}$ & $f_{x}$ & $N_{x} \times N_{y}$ & $f_{x}$ & $N_{x} \times N_{y}$ & $f_{x}$ \\
\hline Block I & $40 \times 20$ & 0.9121 & $80 \times 40$ & 0.9554 & $160 \times 80$ & 0.9776 \\
\hline Block II & $100 \times 20$ & 1.0370 & $200 \times 40$ & 1.0183 & $400 \times 80$ & 1.0091 \\
\hline Block III & $100 \times 30$ & 1.0370 & $200 \times 60$ & 1.0183 & $400 \times 120$ & 1.0091 \\
\hline Block IV & $100 \times 30$ & 1.0370 & $200 \times 60$ & 1.0183 & $400 \times 120$ & 1.0091 \\
\hline $\mathrm{NC}$ & \multicolumn{2}{|c|}{8800} & \multicolumn{2}{|c|}{35200} & \multicolumn{2}{|c|}{140800} \\
\hline \multirow[t]{2}{*}{ DOF } & \multicolumn{2}{|c|}{52800} & \multicolumn{2}{|c|}{211200} & \multicolumn{2}{|c|}{844800} \\
\hline & \multicolumn{2}{|c|}{$\Delta x_{\min }=\Delta y_{\min }=0.05$} & \multicolumn{2}{|c|}{$\Delta x_{\min }=\Delta y_{\min }=0.025$} & \multicolumn{2}{|c|}{$\Delta x_{\min }=\Delta y_{\min }=0.0125$} \\
\hline
\end{tabular}




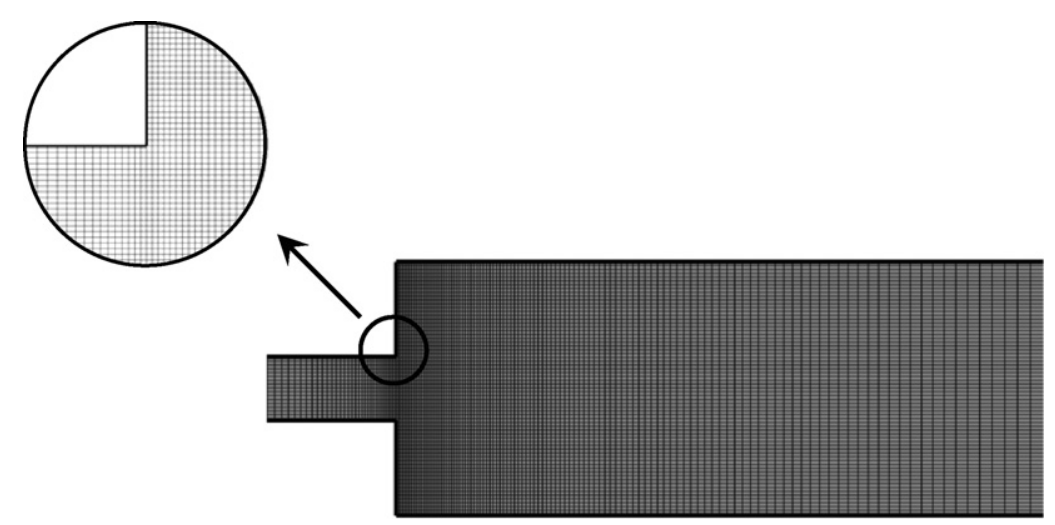

Fig. 3. Zoomed view of the medium mesh used in the computational calculation $(-2 \leq x \leq 10 ;-2 \leq y \leq 2)$.

son's extrapolation" are given in Table 2, for the vortices sizes $\left(X_{\mathrm{r}}\right)$ and intensities $\left(\psi_{\mathrm{r}}\right)$.

The discretisation errors on Mesh 2 (our base mesh for the remaining results) are also given in the previous table. It can seen that the discretisation errors for the recirculation size are below $0.15 \%$ for the Newtonian fluid and $1.4 \%$ for the viscoelastic fluid, while errors in recirculation intensity are below $0.7 \%$ for the Newtonian fluid and $10.3 \%$ for the FENE-MCR simulations. The errors in $\psi_{\mathrm{r}}$ are much higher than errors in $X_{\mathrm{r}}$ because the evaluation of $\psi_{\mathrm{r}}$ requires integration of the resulting velocity fields and this integration tends to lower the accuracy of the results (see Alves et al. [20]).

Thus, in general, the uncertainty in our directly calculated values is around $1-2 \%$ and the stress fields, in particular, which are so important for the correct prediction of viscoelastic flows, were observed to converge well with mesh refinement. For the viscoelastic case in Table 2 (i.e. part (b)) a detailed view of contour plots of $\tau_{x x}$ predicted on the three meshes, not shown here for conciseness, confirms that the results of the two finer meshes are almost coincident.

\section{Results and discussion}

In this section we present and discuss our results and, from the point of view of their practical utility, they may be classified as

Table 2

Effect of mesh refinement for $R e=20$

\begin{tabular}{lll}
\hline & $X_{\mathrm{r}}$ & $\psi_{\mathrm{r}}\left(\times 10^{-2}\right)$ \\
\hline (a) Newtonian case & & \\
Mesh 1 & 3.6281 & 6.5039 \\
Mesh 2 & 3.6307 & 6.5487 \\
Mesh 3 & 3.6349 & 6.5827 \\
Richardson's extrapolation & 3.6363 & 6.5940 \\
Discretisation error (\%) & 0.15 & 0.69 \\
(b) Viscoelastic case & & \\
Mesh 1 & 2.0684 & 2.2307 \\
Mesh 2 & 2.1562 & 2.6163 \\
Mesh 3 & 2.1787 & 2.8421 \\
Richardson's extrapolation & 2.1862 & 2.9174 \\
Discretisation error $(\%)$ & 1.37 & 10.32 \\
\hline
\end{tabular}

$W e=2, \beta=0.5$ and $L^{2}=100$. either qualitative or quantitative results. The qualitative results to be given essentially comprise streamline plots, an effective way of illustrating the effect of inertia, elasticity and solvent viscosity ratio on the degree of recirculation and observing the existence, or not, of asymmetric flow. In addition the main contribution of the work is however the quantitative analysis, which comprises tables and figures for the size and intensity of the corner vortex, stress distributions, velocity profiles and the pressure drop along the centreline. Our numerical values for the Newtonian and viscoelastic fluid flows are also compared with the results obtained by Oliveira [11] for a planar sudden expansion with a lower expansion ratio of 3 . Firstly we document our results for the Newtonian case with the main purpose of validating the calculations. An equivalent validation could not be undertaken for the viscoelastic simulations because we could not find an adequate data set in the literature for comparison. Next we consider the viscoelastic case, for which we study the effects of elasticity, polymer concentration and inertia. In the current work $L^{2}$ remains constant, at value of $L^{2}=100$, in accordance with the work of Remmelgas et al. [22]. Section 5.2.3 discusses briefly the influence of extensibility, in the range $L^{2}=100-500$.

All calculations presented in this work were conducted using a Pentium ${ }^{\circledR}$ IV personal computer with $3.0 \mathrm{GHz}$ clock speed and $1024 \mathrm{MB}$ random access memory. The computational time was seen to increase almost linearly with the mesh density (number of cells).

\subsection{Results for the Newtonian case (validation)}

In Fig. 4 we compare our predicted bifurcation results with the numerical values obtained in the work of Drikakis [2]. Following Drikakis we find it useful to define the parameter DX, where $\mathrm{DX}=\left(X_{\mathrm{r} 1}-X_{\mathrm{r} 2}\right)$, to quantify the existence, or not, of asymmetric flow. The parameter DX is zero for a symmetric flow and non-zero, with opposite signs, for the two possible asymmetric flow states after the critical Reynolds number. The upper branch in Fig. 4 corresponds to flow attaching first to the upper wall and, conversely, the lower branch corresponds to the flow attaching to the lower wall. Drikakis analysed Newtonian flow in several expansion ratios, imposing fully-developed conditions at inlet and defining the Reynolds number using the maximum inlet velocity $\left(U_{0}=1.5 U\right)$ and height of the inlet channel $(d)$. 


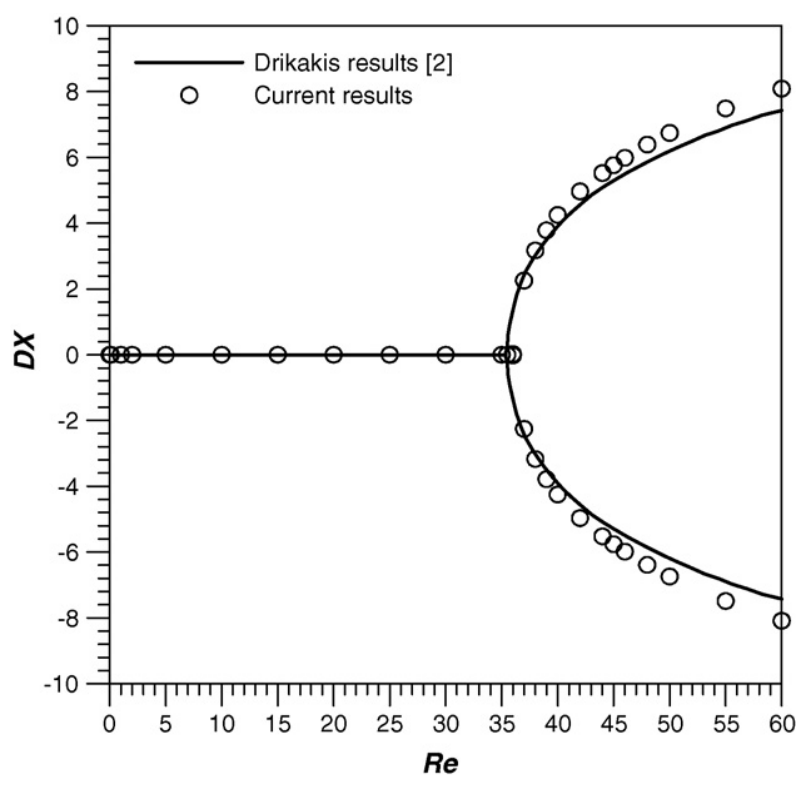

Fig. 4. Comparison of the bifurcation parameter DX between our simulations and the results of Drikakis [2] results for the Newtonian fluid.

We have reprocessed his results so the definitions used in Fig. 4 are consistent with ours. Our results are, in the main, in good agreement with the results of Drikakis except for a discernable systematic difference for $R e>45$.

Our predicted results for a Newtonian fluid are also presented in Table 3 . The corner vortex size is measured by its non-dimensional length, $X_{\mathrm{r}}=x_{\mathrm{r}} / d$, and the intensity is calculated by the amount of recirculating flow normalised by the inlet flow

Table 3

Predicted vortex data for the Newtonian fluid

\begin{tabular}{rrlllll}
\hline$R e$ & $X_{\mathrm{r} 1}$ & $X_{\mathrm{r} 2}$ & $X_{\mathrm{r} 3}$ & $X_{\mathrm{r} 4}$ & $\psi_{\mathrm{rl}}\left(\times 10^{-2}\right)$ & $\psi_{\mathrm{r} 2}\left(\times 10^{-2}\right)$ \\
\hline 0.01 & 0.747 & 0.747 & - & - & 0.059 & 0.059 \\
0.1 & 0.755 & 0.755 & - & - & 0.062 & 0.062 \\
1 & 0.837 & 0.837 & - & - & 0.103 & 0.103 \\
2 & 0.937 & 0.937 & - & - & 0.173 & 0.173 \\
5 & 1.291 & 1.291 & - & - & 0.672 & 0.672 \\
10 & 1.994 & 1.994 & - & - & 2.480 & 2.480 \\
20 & 3.631 & 3.631 & - & - & 6.549 & 6.549 \\
30 & 5.374 & 5.374 & - & - & 8.812 & 8.812 \\
35 & 6.262 & 6.262 & - & - & 9.483 & 9.483 \\
36 & 6.449 & 6.428 & - & - & 9.597 & 9.589 \\
37 & 7.645 & 5.389 & - & - & 10.15 & 9.345 \\
39 & 8.595 & 4.812 & - & - & 10.86 & 9.282 \\
40 & 8.929 & 4.681 & - & - & 11.20 & 9.290 \\
45 & 10.18 & 4.423 & - & - & 13.28 & 9.396 \\
50 & 11.13 & 4.386 & - & - & 15.28 & 9.512 \\
55 & 11.90 & 4.417 & - & - & 16.81 & 9.607 \\
60 & 12.56 & 4.474 & - & - & 17.99 & 9.672 \\
63 & 12.91 & 4.515 & - & - & 18.57 & 9.700 \\
64 & 13.02 & 4.529 & 13.48 & 14.05 & 18.74 & 9.711 \\
65 & 13.13 & 4.544 & 13.00 & 14.87 & 18.91 & 9.720 \\
70 & 13.61 & 4.619 & 12.51 & 16.87 & 19.64 & 9.745 \\
80 & 14.41 & 4.774 & 12.48 & 19.81 & 20.76 & 9.761 \\
90 & 15.07 & 4.926 & 12.72 & 22.27 & 21.76 & 9.746 \\
100 & 15.69 & 5.075 & 13.06 & 24.56 & 22.87 & 9.703 \\
\hline & & & & & &
\end{tabular}

rate so that, along the cross-stream direction, $\psi_{\mathrm{r}}$ varies from 0 to 1 .

Comparison of our $X_{\mathrm{r}}$ and $\psi_{\mathrm{r}}$ values at $R e=0.01(W e=0)$ with "benchmark" creeping flow calculations of Alves et al. [23] shows agreement of better than $0.5 \%$. Additionally, excellent agreement is obtained with the correlations of Scott et al. [24] in their range of validity (say $20 \leq R e \leq 37$ ). For low Reynolds number, a comparison can also be performed against the results for the 4:1:4 constriction geometry of Cartalos and Piau [25], Szabo et al. [26] and Rothstein and McKinley [27]. These authors have studied experimentally [25,27] and numerically [26] the viscoelastic flow of Boger fluids in axisymmetric contraction/expansions, with particular emphasis placed on the flow upstream of the first contraction. For low Deborah numbers the results are almost independent of whether the flow is nonNewtonian or not, and whether it occurs in a contraction or an expansion; in that situation [27] measured a vortex length and the coordinates of the vortex central position which compares very well with our predictions at $D e=1$.

The effect of Reynolds number on the pressure distribution along the "lower" and "upper" wall is shown in Fig. 5 for the Newtonian fluid at Reynolds numbers equal to 36, 45, 70 and 100. (Note in this work the larger recirculation is always shown as occurring on the lower wall and the smaller on the upper wall, but in the simulations it was equally likely to occur on either wall and is only plotted as such for consistency.) Under symmetric separation conditions, e.g. $R e=36$ in Fig. 5 (a condition just subcritical), the pressure distribution on the lower and upper wall are the same (the continuous and dashed lines are undistinguishable). Downstream of the sudden expansion the pressure initially increases (pressure recovery after the increase in channel cross-section), reaches a maximum value, and then gradually decreases toward the outlet of the channel. Predictions of Drikakis [2] are also shown in the figure and good agreement is observed; our predicted slope for the pressure decay is 0.33329 in the range $x / d=20$ and 40, comparing extremely well with the theoretical value of $f=12 / R e=0.33333$. When the flow is asymmetric the pressure has different values on the lower and upper walls before eventually reaching the same values and the constant slope for fully developed conditions. At some Reynolds number (e.g. $R e>70$, see Fig. 15(a) for the streamline patterns in these cases) a pressure drop occurs downstream of the recirculation region on the lower wall and downstream of the smaller recirculation on the upper wall, but this behaviour does not occur at the farthest recirculation zone for $R e>64$ (see Fig. 15(a) for $R e=70)$ on the upper wall. In this case the recirculation creates a displacement of the flow, seen from the streamlines in Fig. 15(a), and consequently a pressure drop on the opposite wall. However, this does not occur downstream of the large recirculation on the upper wall because the flow on the lower wall is already attached and, therefore, cannot create a flow displacement with an attendant pressure drop. Similar conclusions were drawn in the work of Drikakis [2] against whose numerical results we have compared our predictions.

In Fig. 6 we present a detailed comparison of the axial velocity distributions for several $R e$, corresponding to an asymmetric flow situation, at four axial locations situated at $x / d=10,15,20$ 

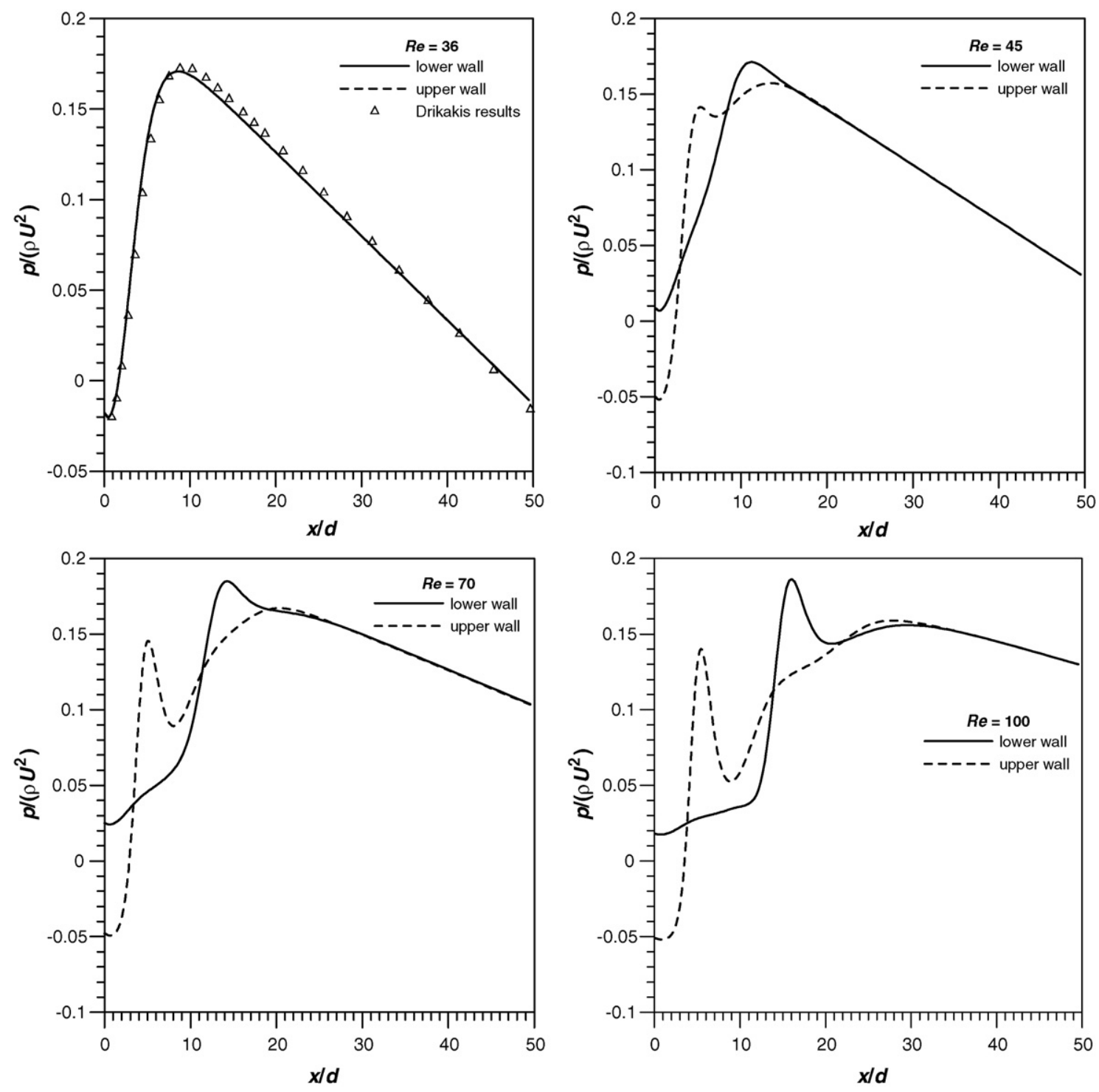

Fig. 5. Pressure distribution at various Reynolds numbers for the Newtonian simulations.

and 25. These locations are illustrated schematically in Fig. 7 which is a streamline plot for $R e=100$ (Newtonian fluid) where a third recirculation region is seen to occur on the upper wall.

It can be seen from the streamline plot, shown in Fig. 7, that at these high Reynolds number an additional recirculation zone has developed downstream of the expansion plane. The flow separates from the upper wall, impinges on the lower wall, and then reattaches to the upper wall as commented above in relation to the pressure distribution. However, Fig. 6 confirms that by $x / d=25$ the flow field has become symmetric once again and is close to the analytical fully developed parabolic profile for all Reynolds numbers.

\subsection{Results for the viscoelastic case}

As already pointed out at the end of Section 2, using the FENE-MCR constitutive equation to model a non-Newtonian viscoelastic fluid the number of independent parameters that can be varied increases from a single parameter in the Newtonian case up to four ( $R e, W e, \beta$ and $L^{2}$ ) and an exhaustive study of all possibilities goes well beyond the present attempt. To investigate their effects, we have decided to fix all parameters except one at some typical values and vary separately the remaining parameter. Such "separated" effects are discussed in this section.

\subsubsection{Effect of elasticity, We number}

To investigate the effect of elasticity we use the Weissenberg number, which provides a relative measure of the magnitude of the elastic to viscous stresses, defined here as $W e=\lambda U / d$. In this case, we only modify the constant relaxation time $\lambda$, which represents a time constant of the material.

Some computational results are provided in Table 4 for the vortices sizes and intensities with increasing We number between 0 and $100\left(\operatorname{Re}=40, \beta=0.9\right.$ and $\left.L^{2}=100\right)$. We note that for this relatively high value of $\beta$ (typical of Boger fluids, cf. Rothstein 



Fig. 6. Comparison of predicted axial velocity profiles for $R e=60,65,80$ and 100 (Newtonian results).

and McKinley [27] who worked with a polystyrene solution having $\beta=0.921$ ) the computations could be pursued up to large Weissenberg numbers, but limiting We values were found at lower $\beta$ (see Section 5.2.2).

In Fig. 8 we present qualitatively the values of Table 4 for the size of the recirculation region as a function of We number, at Reynolds numbers of $R e=40$ and $60\left(\beta=0.9, L^{2}=100\right)$. We can see that the effect of increasing the We number from $W e=0-100$, is for the larger of the two corner vortices $\left(X_{\mathrm{r} 1}\right)$ to be reduced in size, while the shorter vortex $\left(X_{\mathrm{r} 2}\right)$ tends to increase in size in the lower range of $W e$ for $R e=40$, or remains approximately

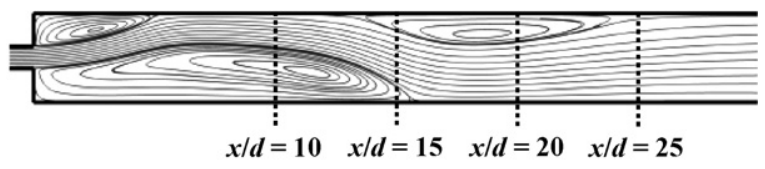

Fig. 7. Schematic illustrating locations of axial-velocity distribution profiles for $x / d=10,15,20$ and 25 (streamlines for $R e=100$ ). constant. However, although the effect of elasticity is to diminish the degree of asymmetry of the flow, the asymmetry is present for the whole range of We number $(0<W e<100)$ studied.

In Fig. 9 we plot the streamlines for We numbers between 0 (Newtonian fluid) and 10, for symmetric flow cases, at a constant Reynolds number of $R e=20\left(\beta=0.5, L^{2}=100\right)$. The effect of elasticity is to reduce the vortex size and the intensity of the recirculating flow in each case. Downstream of the expansion plane a "bulging" of the streamlines can be observed. This phenomenon can be explained if we consider that fluid is converging towards the centreline upstream of the expansion, followed by a stronger divergence (due to elastic recovery of the stretched polymer molecules) towards the larger channel walls after the expansion plane (see Fig. 9 at the highest Weissenberg number, $W e=10$ ).

As a consequence of this convergent-divergent streamline behaviour, an overshoot of the streamwise velocity component along the centreline occurs upstream of the expansion and then 

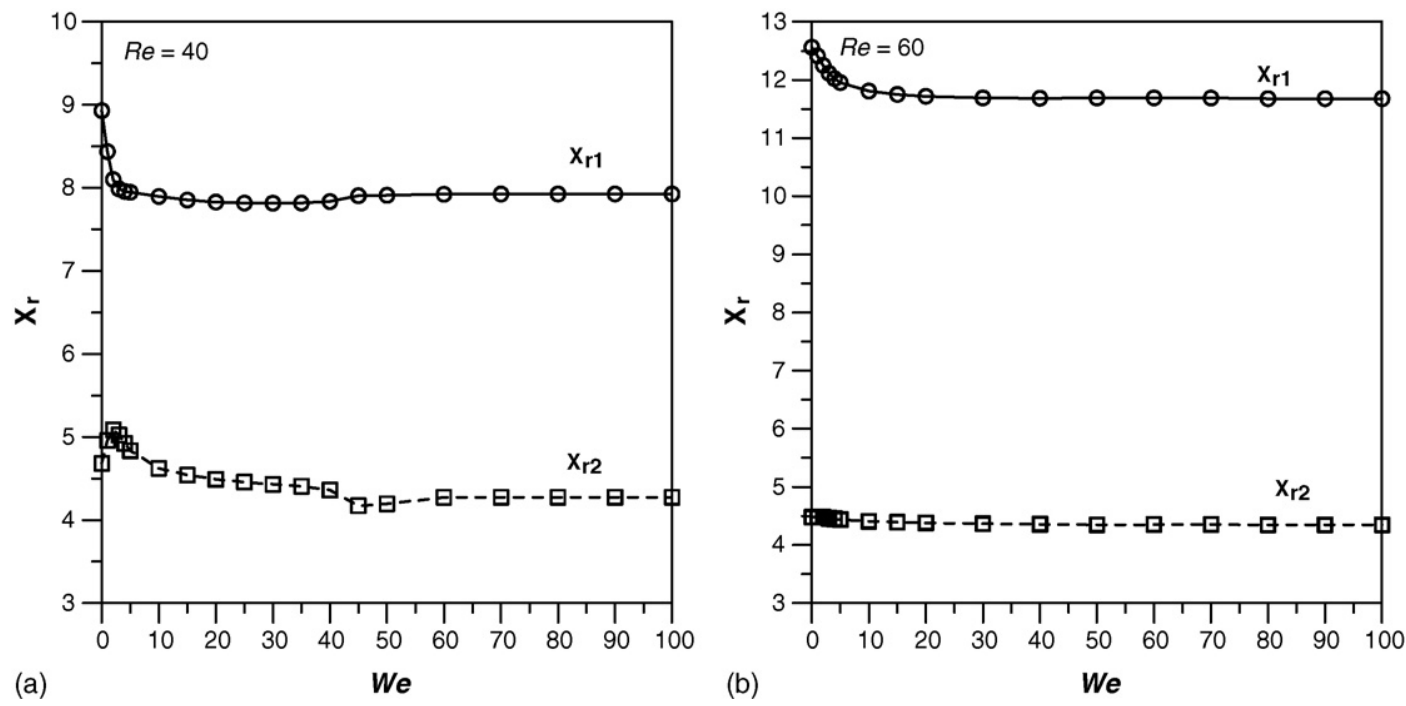

Fig. 8. Effect of Weissenberg number on the size of the two asymmetric vortices $\left(L^{2}=100, \beta=0.9\right)$ : (a) $R e=40$ and (b) $R e=60$.

an undershoot occurs downstream. This behaviour is shown, for the particular case of $R e=20$ and $W e=0$ and $10(\beta=0.5$, $L^{2}=100$ ), in Fig. 10 where it is clear that the fluid acceleration along the centreline is concentrated in a small region just at the entrance to the expansion $(x \approx 0)$, while the region of diverging streamlines is distributed over a longer distance downstream of the expansion plane. The effect becomes more pronounced with increasing $W e$, but is also dependent on the Reynolds number, as shown in Fig. 11 for $W e=2$ and $W e=4$. From this figure, which shows the variation of these velocity over and undershoots along the centreline with $R e$, it is clear that the overshoot of velocity near the expansion plane increases with increasing elasticity number $\left(E=\lambda \eta_{0} / \rho d^{2}=(1-\beta) W e / R e\right)$ and that the undershoot in the diverging streamlines zone reaches a maximum at a given $E$ $(\approx 0.08-0.10)$. A nice and definite explanation for this "diverging flow behaviour" was recently put forth by Alves and Poole [28] for the contraction flow case, but the same mechanism should be at work for the sudden expansion case under consideration here. Their analysis demonstrated that inertia (through the Reynolds numbers) is not necessary for diverging flow to be observed: therefore the phenomenon should not be directly controlled by the elasticity number.

A peculiar feature in the vortex size variation with We seen in Fig. 8 for the lower $R e$ value $(R e=40)$ is worth discussing here because it is an elastic effect that was, in fact, already present in the work of Oliveira [11] (see his Fig. 8) but went unnoticed. There is a slight, but distinct, dip in the size of the smallest vortex $\left(X_{\mathrm{r} 2}\right)$ at $W e=45(R e=40 \Rightarrow E \approx 0.1)$, with an ensuing slight increase of $X_{\mathrm{r} 1}$. In order to clarify the origin of such a small, but discernable 'kink' in the variation of $X_{\mathrm{r}}$, we plot the streamlines
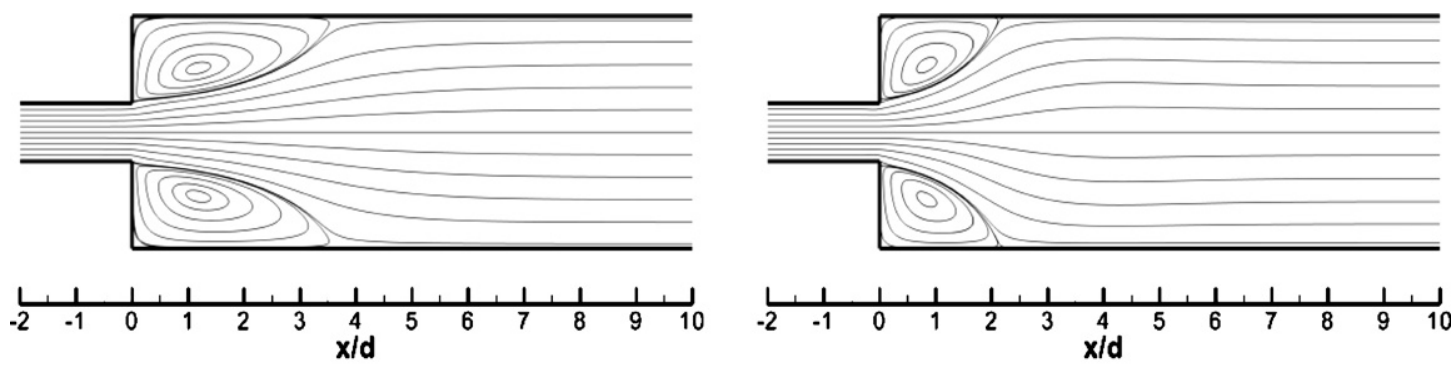

$W e=0$

$W e=2$

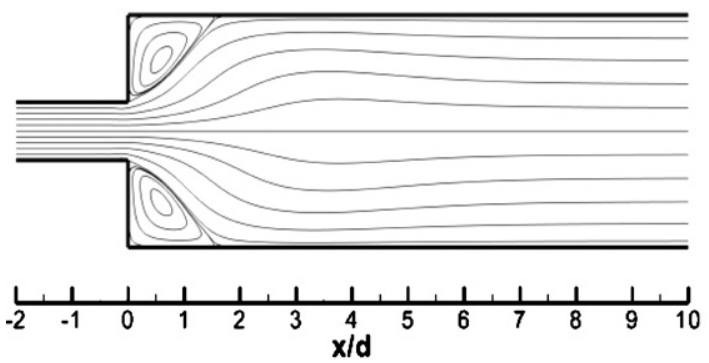

$W e=5$

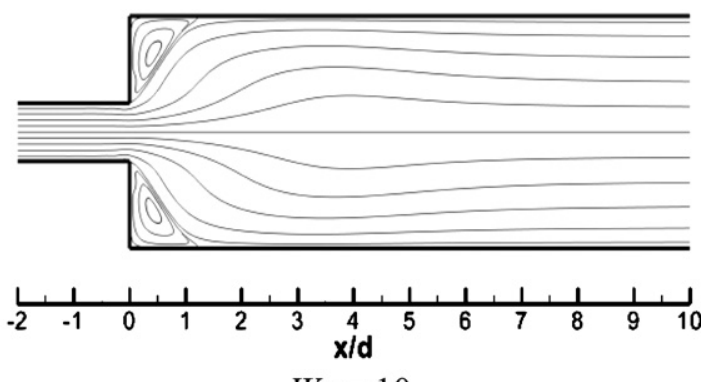

Fig. 9. Streamlines at various We numbers $\left(R e=20, L^{2}=100\right.$, and $\left.\beta=0.5\right)$. 
Table 4

Predicted vortex data for the viscoelastic fluid/Effect of elasticity through We, for $R e=40, \beta=0.9$ and $L^{2}=100$

\begin{tabular}{rllll}
\hline We & $X_{\mathrm{r} 1}$ & $X_{\mathrm{r} 2}$ & $\psi_{\mathrm{r} 1}\left(\times 10^{-2}\right)$ & $\psi_{\mathrm{r} 2}\left(\times 10^{-2}\right)$ \\
\hline 0 & 8.93 & 4.68 & 11.20 & 9.29 \\
1 & 8.44 & 4.96 & 10.50 & 9.05 \\
2 & 8.10 & 5.09 & 10.28 & 8.98 \\
3 & 7.99 & 5.02 & 10.30 & 8.92 \\
4 & 7.96 & 4.92 & 10.38 & 8.85 \\
5 & 7.95 & 4.84 & 10.45 & 8.78 \\
10 & 7.90 & 4.62 & 10.60 & 8.52 \\
15 & 7.85 & 4.54 & 10.59 & 8.40 \\
20 & 7.83 & 4.49 & 10.55 & 8.36 \\
30 & 7.82 & 4.43 & 10.49 & 8.50 \\
40 & 7.83 & 4.36 & 10.44 & 8.78 \\
50 & 7.91 & 4.20 & 10.16 & 9.66 \\
60 & 7.92 & 4.27 & 10.11 & 9.80 \\
70 & 7.92 & 4.28 & 10.10 & 9.91 \\
80 & 7.93 & 4.28 & 10.10 & 9.92 \\
90 & 7.93 & 4.28 & 10.10 & 9.92 \\
100 & 7.93 & 4.28 & 10.10 & 9.92 \\
\hline
\end{tabular}

for the $R e$ of interest in Fig. 12. It appears that for $W e \geq 45$ $(E \geq 0.11)$ the shape of the smaller vortex near the wall changes abruptly from an elongated shape, typical of the Newtonian vortex (cf. $W e=0$ ), to a more strongly convex shape, with the separation streamline now intersecting the wall at a right angle. We hypothesise that this elastic retraction of the smaller vortex is related to the "diverging streamlines" phenomenon discussed above. For $R e=60(W e=2, \beta=0.9, E=(1-0.9) \times 2 / 60=0.003)$ such a perturbation in vortex size is absent, as seen in Fig. 8(b).

\subsubsection{Effect of concentration, $\beta$}

The $\beta$ parameter measures the ratio of solvent viscosity to total shear viscosity. For the present study we varied $\beta$ from 1.0 to 0.3 , at fixed $R e=40, W e=2$ and $L^{2}=100$.

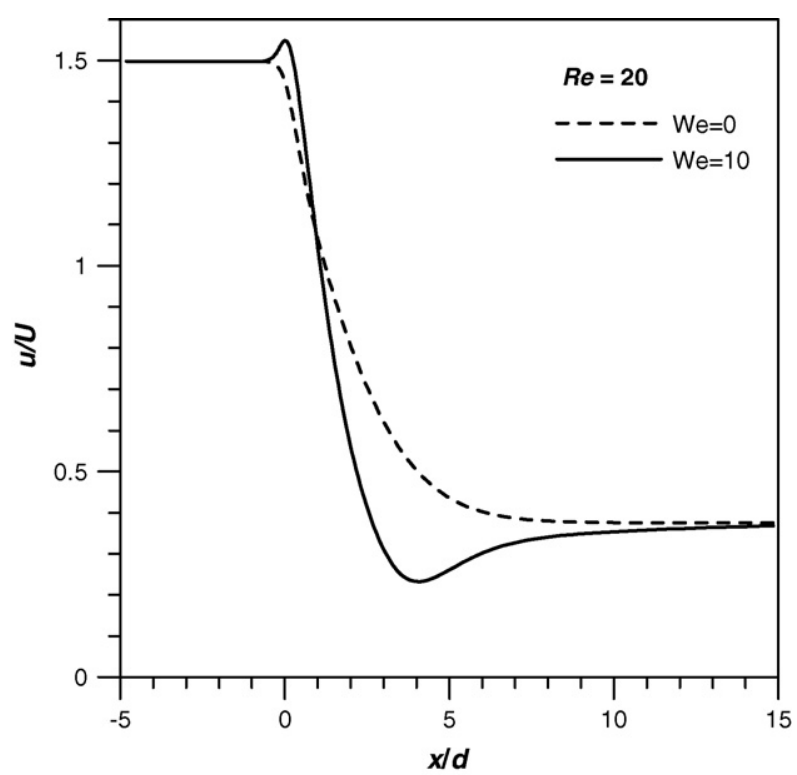

Fig. 10. Variation of streamwise velocity component along the centerline for $R e=20$ at $W e=0$ (Newtonian fluid) and $W e=10$ (viscoelastic fluid, $\beta=0.5$ and $\left.L^{2}=100\right)$.

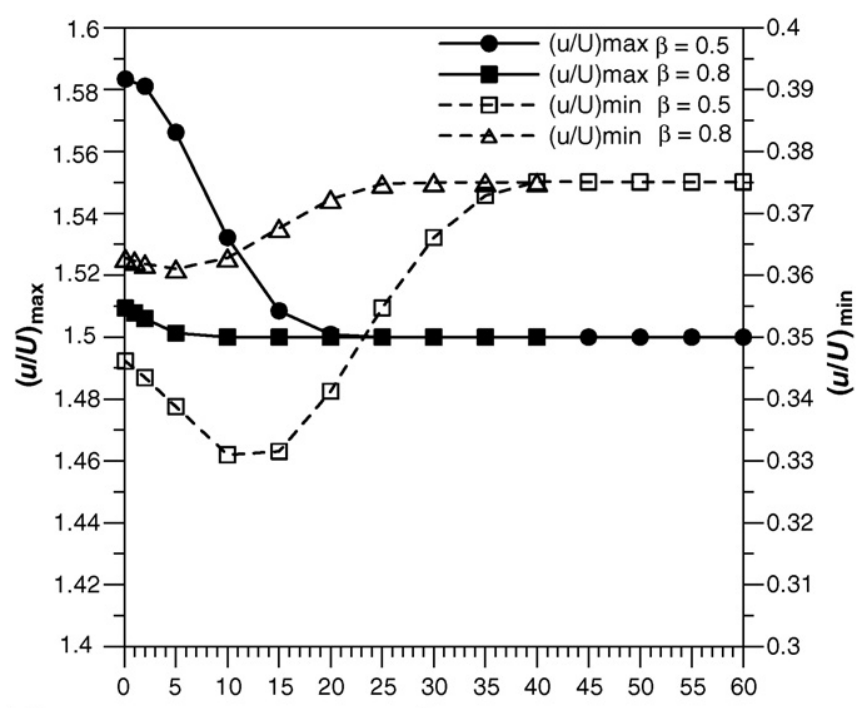

(a)

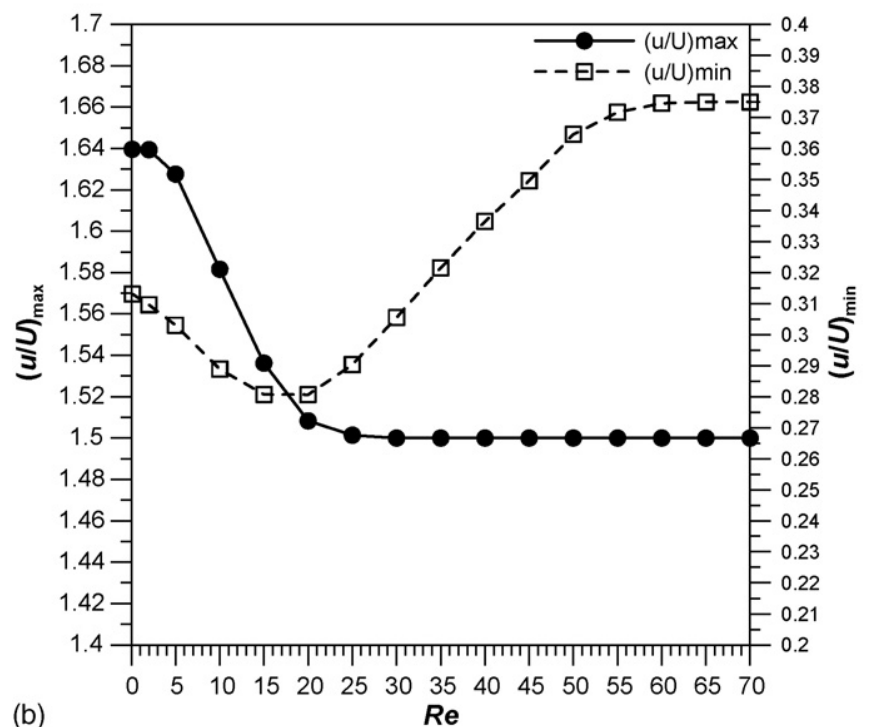

Fig. 11. Variation of the velocity overshoot and undershoot with Reynolds number for two Weissenberg numbers $\left(L^{2}=100\right)$ : (a) $W e=2(\beta=0.5$ and 0.8$)$ and (b) $W e=4(\beta=0.5)$.

The numerical results of the present parametric study, in terms of vortex size and intensity, are given in Table 5 and qualitatively illustrated in Fig. 13.

Analysing Fig. 13 we can see that for a decreasing $\beta$ ratio the flow is stabilized until a stable symmetrical state exists

Table 5

Predicted vortex data for the viscoelastic fluid/Effect of concentration parameter $\beta$, for $W e=2, \operatorname{Re}=40$ and $L^{2}=100$

\begin{tabular}{lllll}
\hline$\beta$ & $X_{\mathrm{r} 1}$ & $X_{\mathrm{r} 2}$ & $\psi_{\mathrm{r} 1}\left(\times 10^{-2}\right)$ & $\psi_{\mathrm{r} 2}\left(\times 10^{-2}\right)$ \\
\hline 1.0 & 8.929 & 4.681 & 11.20 & 9.290 \\
0.9 & 8.101 & 5.086 & 10.28 & 8.983 \\
0.8 & 6.644 & 6.129 & 9.189 & 8.967 \\
0.7 & 5.993 & 6.059 & 8.601 & 8.639 \\
0.6 & 5.659 & 5.693 & 8.158 & 8.178 \\
0.5 & 5.319 & 5.336 & 7.662 & 7.671 \\
0.4 & 4.979 & 4.989 & 7.035 & 7.041 \\
0.3 & 4.651 & 4.659 & 6.321 & 6.325 \\
\hline
\end{tabular}




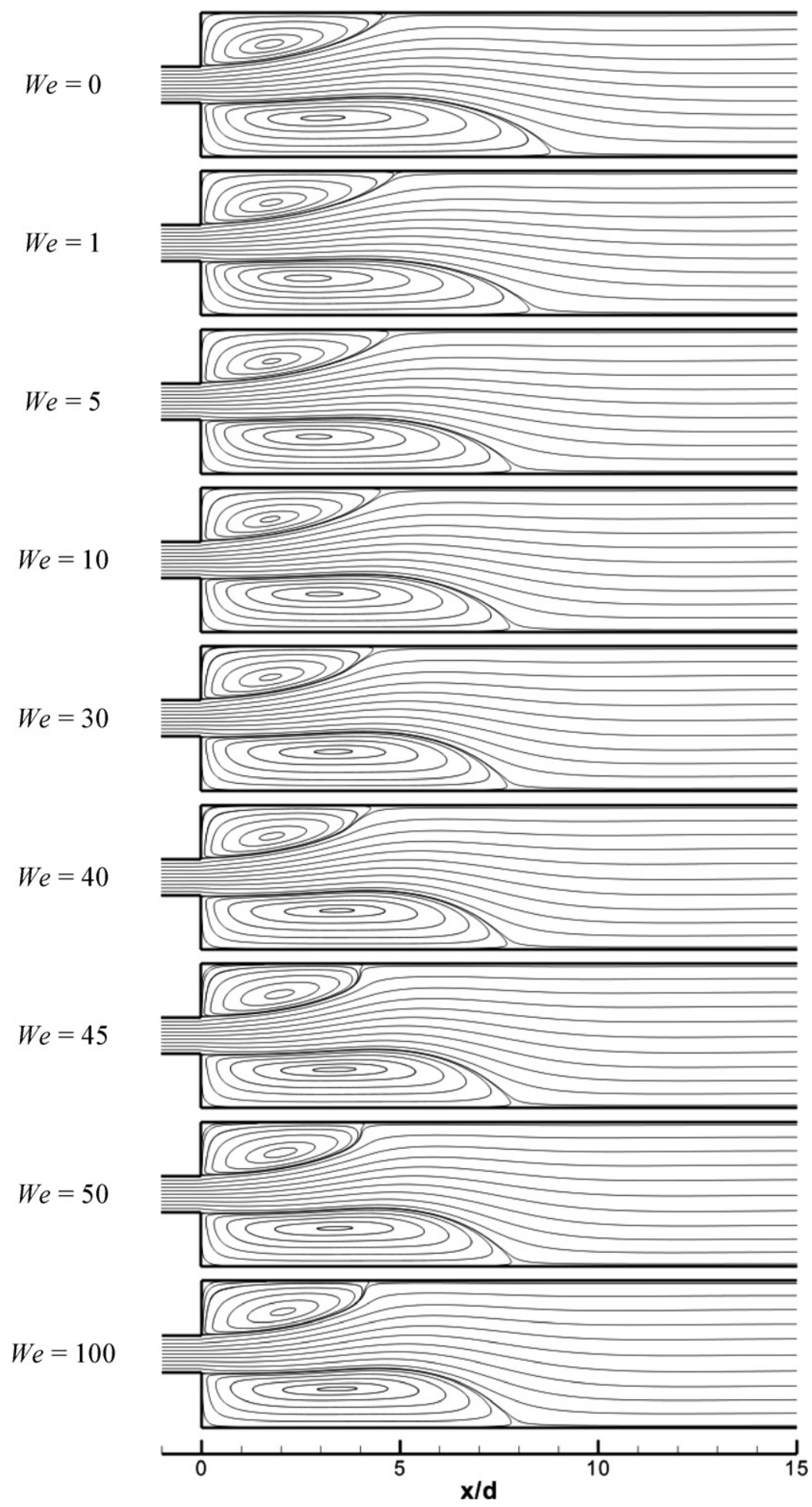

Fig. 12. Vortex shapes at increasing Weissenberg number $\left(\beta=0.9\right.$ and $\left.L^{2}=100\right)$ and constant Reynolds number $(R e=40)$. Note change at $W e=45$.

for values of $\beta \leq 0.8$, at $R e=40$, while the flow is asymmetric for $\beta>0.8$. It is clear that the Newtonian flow $(\beta=1.0)$ has the largest asymmetry and this asymmetry is somewhat reduced with a small introduction of elasticity in the fluid, through the $\beta$ parameter, and it is completely attenuated when the concentration is further increased. Following the practice of defining the Weissenberg number based on a Maxwell model relaxation time (that is, $\lambda_{0}=\psi_{1} / 2 \eta_{0}$, where $\psi_{1}$ is the first normal-stress coefficient), the influence of $\beta$ can be seen as an elastic effect provided we use $W e^{\prime}=W e(1-\beta)$ to define the Weissenberg number. For $\beta=1$ we have $W e^{\prime}=0$ and no influence of viscoelasticity is expected; for $\beta<1$, with progressively lower values, $W e^{\prime}$ increases implying higher levels of elasticity. 


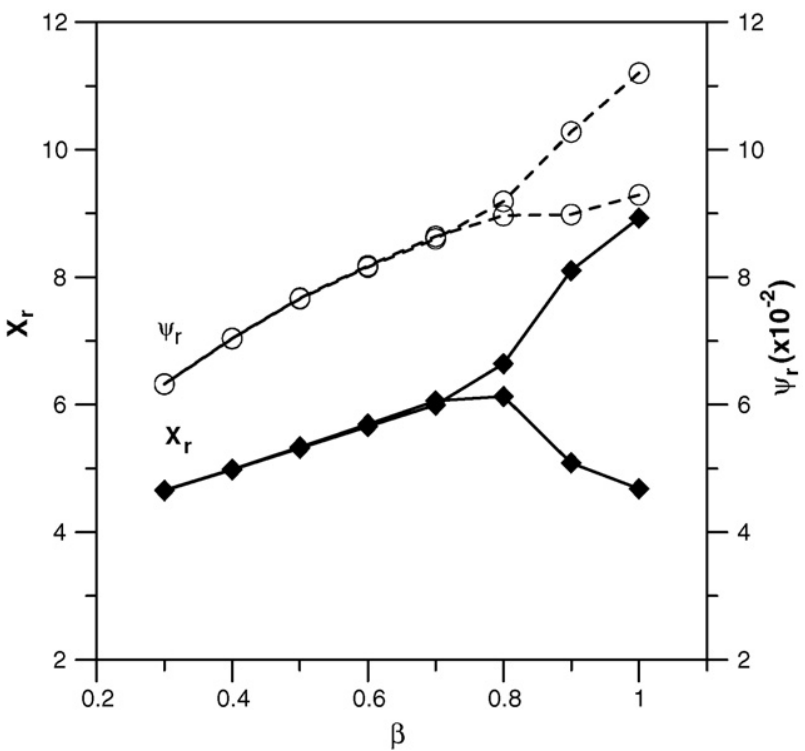

Fig. 13. Effect of solvent concentration $\left(\beta=\eta_{\mathrm{s}} / \eta_{0}\right)$ upon size (filled symbols) and intensity (hollow symbols) of vortices $\left(R e=40, W e=2\right.$, and $\left.L^{2}=100\right)$.

Table 6

Predicted vortex data for the viscoelastic fluid/Effect of extensibility parameter $L^{2}$, for $R e=40, \beta=0.9$ and $W e=2$

\begin{tabular}{lllll}
\hline$L^{2}$ & $X_{\mathrm{r} 1}$ & $X_{\mathrm{r} 2}$ & $\psi_{\mathrm{r} 1}\left(\times 10^{-2}\right)$ & $\psi_{\mathrm{r} 2}\left(\times 10^{-2}\right)$ \\
\hline 100 & 8.101 & 5.086 & 10.28 & 8.983 \\
200 & 7.945 & 5.287 & 9.858 & 8.731 \\
300 & 7.899 & 5.383 & 9.605 & 8.525 \\
400 & 8.038 & 5.210 & 9.558 & 8.256 \\
500 & 8.208 & 4.973 & 9.537 & 7.975 \\
\hline
\end{tabular}

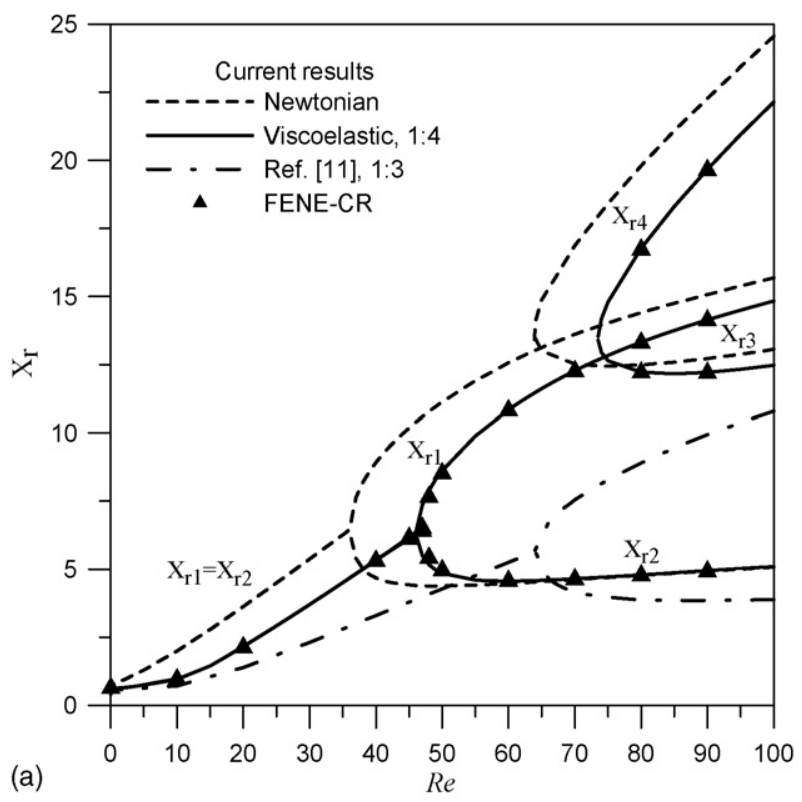

Table 7

Predicted vortex data for the viscoelastic fluid ( $W e=2, \beta=0.5$ and $L^{2}=100$ )

\begin{tabular}{lllllll}
\hline$R e$ & $X_{\mathrm{r} 1}$ & $X_{\mathrm{r} 2}$ & $X_{\mathrm{r} 3}$ & $X_{\mathrm{r} 4}$ & $\psi_{\mathrm{r} 1}\left(\times 10^{-2}\right)$ & $\psi_{\mathrm{r} 2}\left(\times 10^{-2}\right)$ \\
\hline 0.01 & 0.605 & 0.619 & - & - & 0.024 & 0.026 \\
0.1 & 0.607 & 0.621 & - & - & 0.024 & 0.027 \\
5 & 0.749 & 0.747 & - & - & 0.062 & 0.062 \\
10 & 0.957 & 0.955 & - & - & 0.188 & 0.187 \\
20 & 2.156 & 2.158 & - & - & 2.616 & 2.615 \\
30 & 3.703 & 3.708 & - & - & 5.863 & 5.868 \\
40 & 5.316 & 5.331 & - & - & 7.677 & 7.686 \\
42 & 5.646 & 5.666 & - & - & 7.942 & 7.954 \\
44 & 5.970 & 6.006 & - & - & 8.179 & 8.200 \\
45 & 6.128 & 6.166 & - & - & 8.286 & 8.310 \\
46 & 6.345 & 6.296 & - & - & 8.426 & 8.399 \\
47 & 7.363 & 5.490 & - & - & 9.179 & 8.340 \\
48 & 7.891 & 5.161 & - & - & 9.705 & 7.942 \\
50 & 8.627 & 4.849 & - & - & 10.65 & 7.884 \\
60 & 10.84 & 4.564 & - & - & 14.57 & 8.022 \\
70 & 12.27 & 4.651 & - & - & 17.01 & 8.198 \\
73 & 12.62 & 4.691 & - & - & 17.54 & 8.239 \\
73.5 & 12.67 & 4.698 & 13.39 & 13.53 & 17.62 & 8.245 \\
74 & 12.73 & 4.705 & 12.94 & 14.18 & 17.69 & 8.250 \\
75 & 12.83 & 4.719 & 12.64 & 14.80 & 17.85 & 8.264 \\
80 & 13.32 & 4.794 & 12.22 & 16.77 & 18.53 & 8.313 \\
85 & 13.75 & 4.871 & 12.15 & 18.30 & 19.09 & 8.352 \\
90 & 14.14 & 4.948 & 12.21 & 19.65 & 19.57 & 8.384 \\
100 & 14.83 & 5.100 & 12.47 & 22.15 & 20.55 & 8.418 \\
\hline
\end{tabular}

At the particular values of Weissenberg number and extensibility parameter of Table 5, it was observed that any further reduction of $\beta$ would result in either a lack of convergence of our iterative scheme or plain divergence. This limit on the allowable parameter range can be due to a deficiency of the numerical method or, alternatively, to the fact that physically the flow becomes unsteady or three-dimensional.

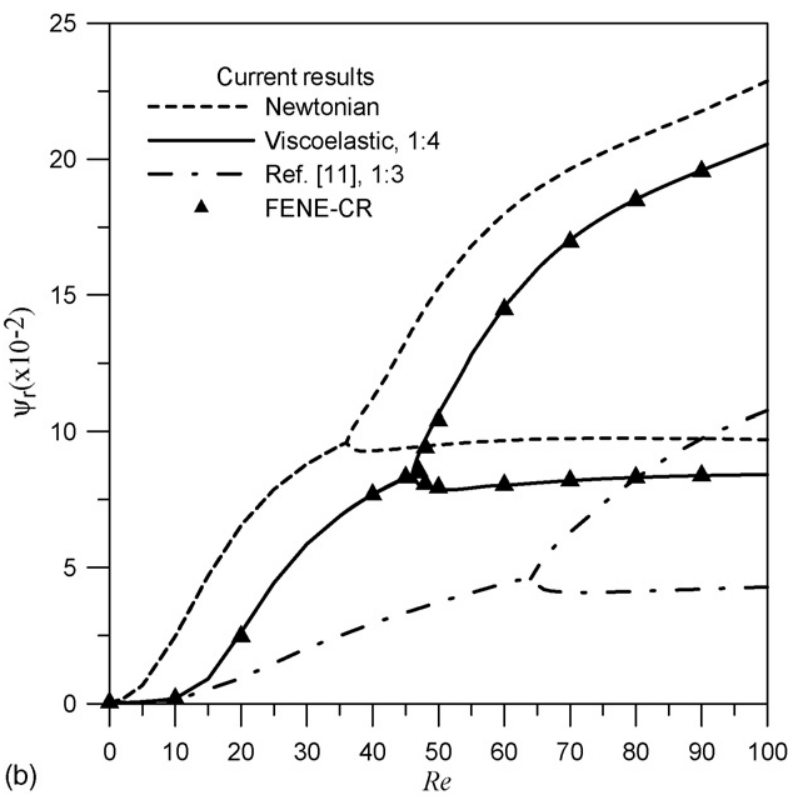

Fig. 14. Comparison of the predicted variation of vortex characteristics with the Reynolds number for the Newtonian and viscoelastic fluids $($ We $=2, \beta=0.5$ and $L^{2}=100$ ), and the viscoelastic results of Oliveira [11] for the 1:3 expansion: (a) vortex size and (b) vortex intensity. New FENE-CR results are shown by a triangular symbol. 


\subsubsection{Influence of extensibility, $L^{2}$}

As mentioned previously, $L^{2}=100$ is a typical value for dilute polymer solutions, already allowing for considerable molecular extension before the fully stretched state is attained. It is interesting however, at least from the point of view of
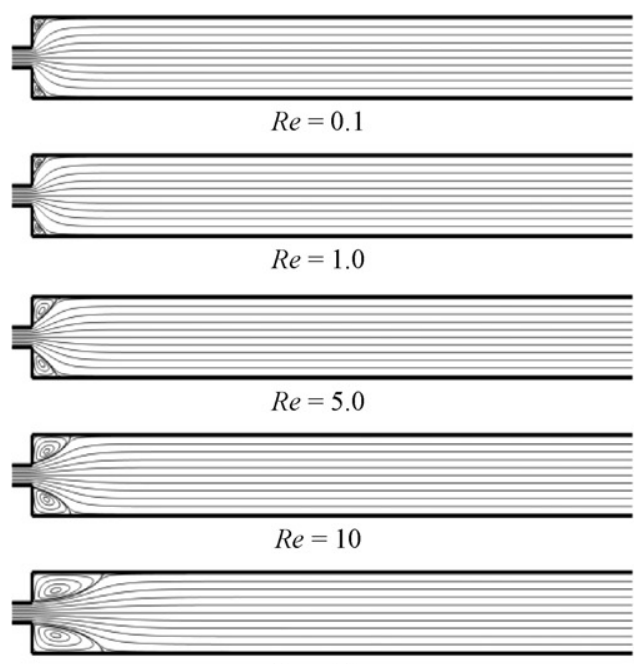

$R e=20$
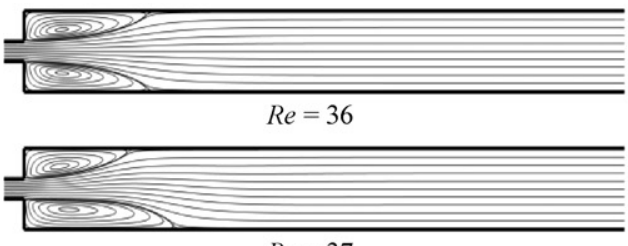

$\operatorname{Re}=37$

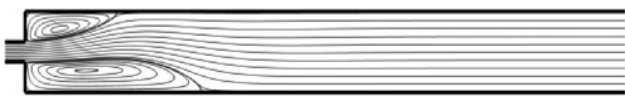

$\operatorname{Re}=40$
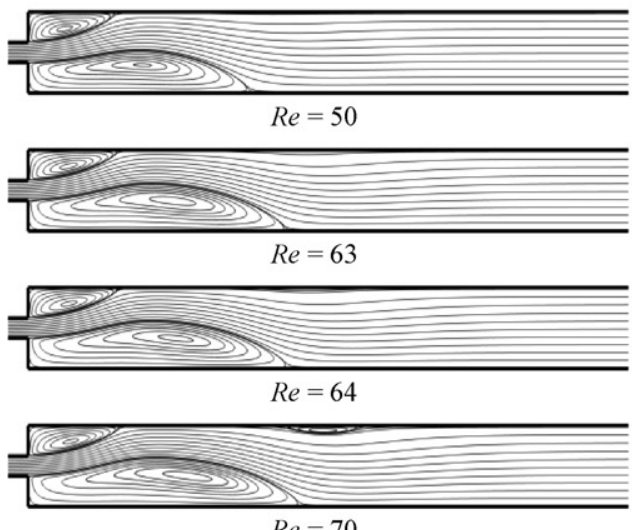

$R e=70$
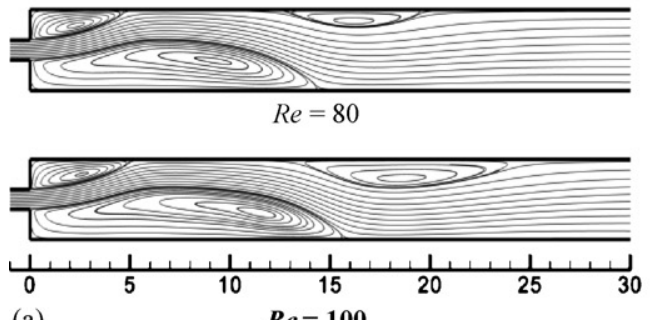

(a)

$R e=100$ completeness, to assess the importance of this level for the $L^{2}$ model parameter on the prediction of vortex sizes. For that, we took the base case of Section 5.2.2 with $R e=40, W e=2$ and $\beta=0.9$, a situation for which there is already bifurcation with two asymmetric vortices, and varied $L^{2}$ from 100 to 500 . The
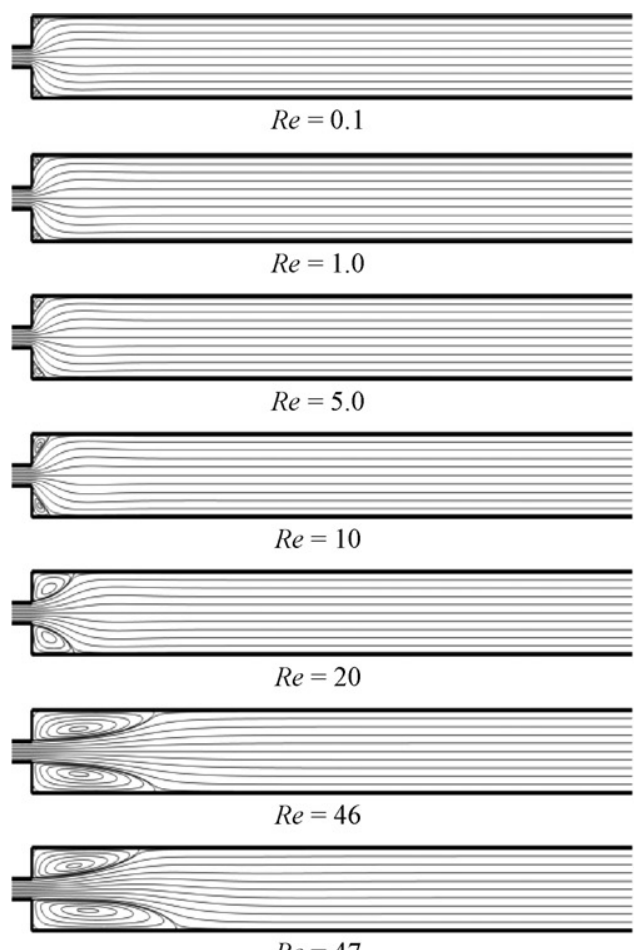

$R e=47$
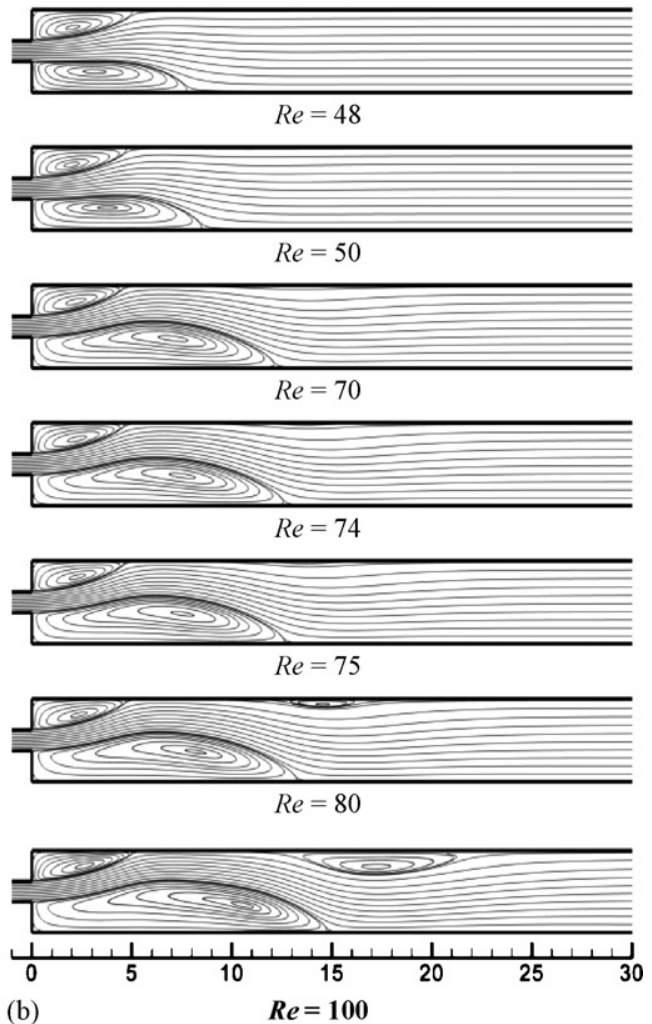

(b)

Fig. 15. Streamlines at various Reynolds numbers: (a) Newtonian fluid and (b) viscoelastic fluid (We $=2, \beta=0.5$ and $L^{2}=100$ ). 


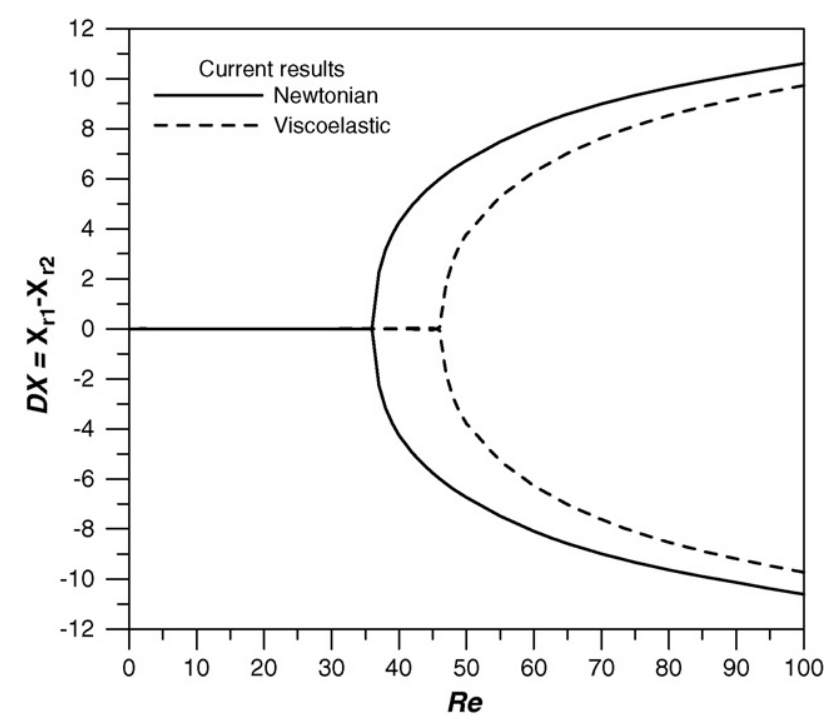

Fig. 16. Bifurcation diagrams for Newtonian and viscoelastic $(W e=2, \beta=0.5$ and $L^{2}=100$ ) flows in a 1:4 planar expansion.

corresponding vortex size and intensity barely varied as seen from Table 6, with a systematic small decrease of the intensity of both vortices, as expected from an increase in extensional elasticity. In conclusion, the influence of $L^{2}$ on the flow, when $L^{2}$ is larger than 100 , appears to be minimal.

\subsubsection{Effect of inertia, Re number}

In this section we present the results concerned with the variation of Reynolds number, for typical values $L^{2}=100$, We $=2$ and a moderate concentration $\beta=0.5$. A Reynolds number measures the ratio between the viscous and inertial forces. At high $R e$ values the flow is dominated by inertial forces and for low $R e$
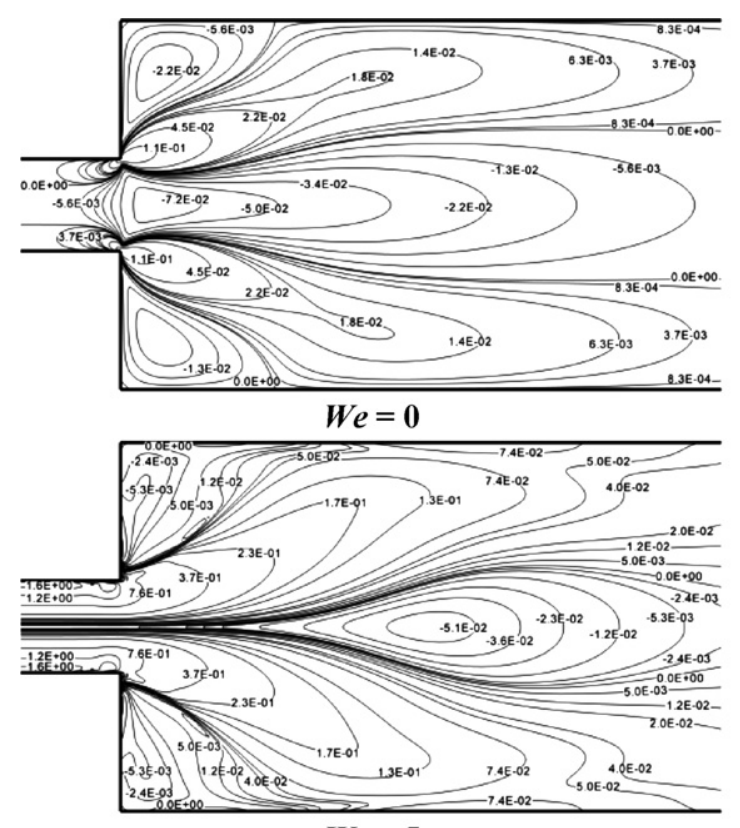

$$
W e=5
$$

values the flow is dominated by viscous forces and the inertial forces are less significant.

Our numerical data are provided in Table 7 and the values are plotted in Fig. 14(a), which shows the variation of the lengths of the upper and lower vortices downstream of the expansion plane with Reynolds number and in Fig. 14(b), which shows the corresponding recirculation intensities. At very low $R e$, some rather small differences are seen in Table 7 between the values of the two, nominally identical, vortex sizes but they are within the numerical uncertainty $(\approx 0.2-2.0 \%)$ of our results. For a Reynolds number up to a critical value $\left(R e_{\mathrm{cr}}=46\right)$ the flow remains steady and symmetric, while for higher $R e$ number the flow is still steady but asymmetric with a larger and a smaller recirculation. For $R e$ above 73.5 a further recirculation region appears on either wall. In Fig. 15 we plot the streamlines for various Reynolds numbers to highlight these effects. Additionally, in Fig. 14 we include the results of [11] for a similar viscoelastic fluid model in a 1:3 expansion to illustrate the effects brought about by a reduction in expansion ratio: essentially a delayed critical bifurcation point and smaller and less intense vortices.

Another issue is whether the modification introduced in the FENE-CR model (MCR against CR, cf. Section 2) produces significant differences. The triangular symbols in Fig. 14 correspond to test calculations performed with the actual CR model and it may be concluded that minimal differences are seen for low to moderate $R e$, up to and around the bifurcation point, and the discrepancies remain small at higher Re. Previous simulations with inertialess flows showed the two models to yield essentially the same results. Thus, the conclusions drawn here for the FENE-MCR model can, in general, be extended to the actual CR version of the FENE model.

In Fig. 16 we show the "bifurcation" plot (i.e. the variation of the DX parameter with $R e$ ) for the viscoelastic
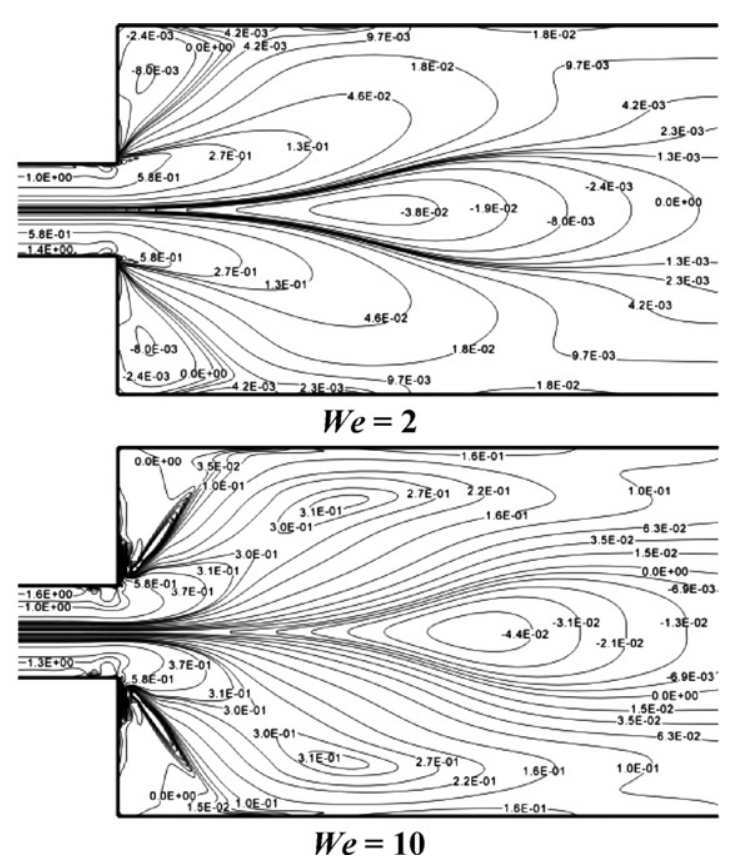

Fig. 17. Non-dimensional contour lines for the first normal-stress differences $\left(N_{1}\right)$ with increasing We numbers $\left(\operatorname{Re}=20, \beta=0.5\right.$ and $\left.L^{2}=100\right)$. 

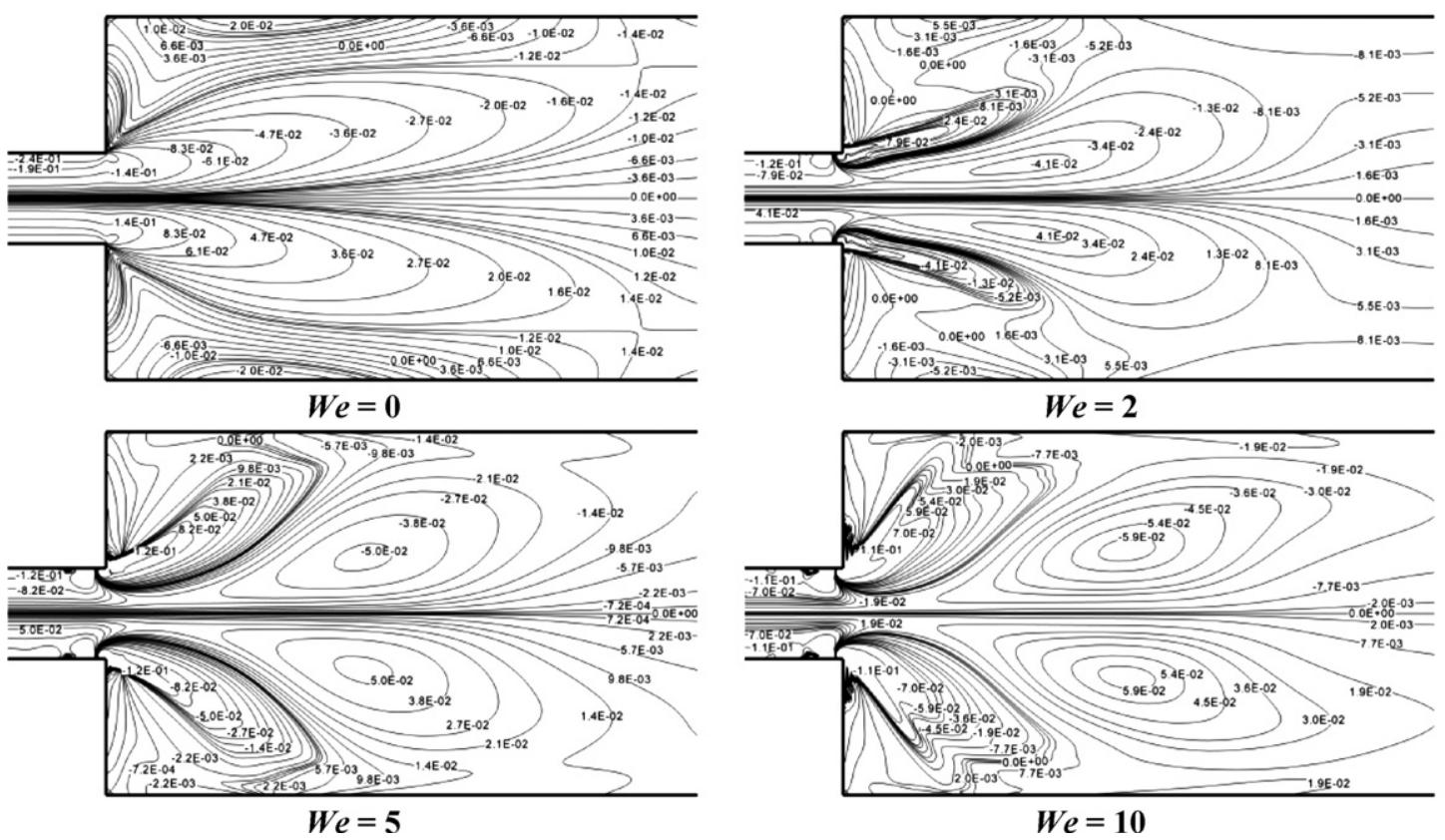

Fig. 18. Non-dimensional contour lines for the shear stress $\left(\tau_{x y}\right)$ with increasing We numbers $\left(R e=20, \beta=0.5\right.$ and $\left.L^{2}=100\right)$.

liquid, and compare to the equivalent data for the Newtonian fluid.

From our results we can conclude that the transition from a symmetric to an asymmetric state is delayed to higher $R e$ values by elasticity, specifically from $R e_{\mathrm{cr}}=36$ (Newtonian case) to $R e_{\mathrm{cr}}=46$ (viscoelastic case) for this parameter set (see Fig. 14). The effect of elasticity is therefore a stabilizing factor for the occurrence of bifurcation, under steady flow conditions. The vortex sizes and intensities are smaller for the viscoelastic liquid when compared with the Newtonian fluid. This effect is observed for the whole range of $R e$, from 0 to 100 , and can be seen in Fig. 15(a and b) which shows the streamline plots for the Newtonian and viscoelastic fluid, respectively. In this figure it is also possible to appreciate the phenomenon of 'diverging' flow commented upon above.

In Figs. 17 and 18 we present the non-dimensional contour lines for the first normal-stress differences $\left(N_{1}=\tau_{x x}-\tau_{y y}\right)$ and shear stress $\left(\tau_{x y}\right)$, with increasing Weissenberg number $(W e=0$, 2, 5 and 10) for $\operatorname{Re}=20\left(\beta=0.5\right.$ and $\left.L^{2}=100\right)$.

Globally these figures illustrate the higher level of stress concentration for the viscoelastic fluid, both in respect of the normal and shear components. The stress profiles are still symmetric,
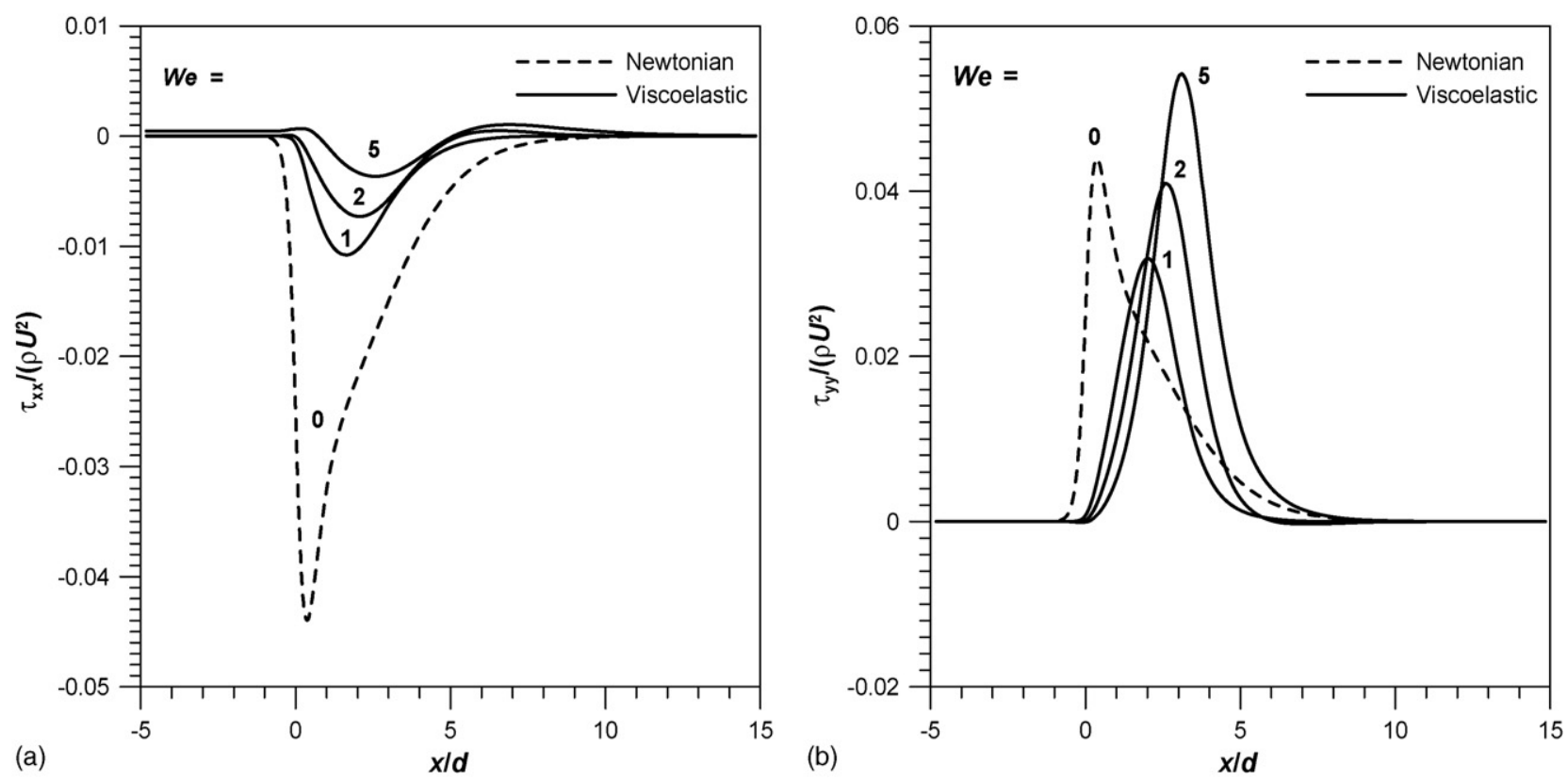

Fig. 19. (a and b) Normal-stress profile $\tau_{x x}$ and $\tau_{y y}$, along the centreline $(y=0)$, for the viscoelastic fluid $\left(\operatorname{Re}=20, \beta=0.5\right.$ and $\left.L^{2}=100\right)$. 

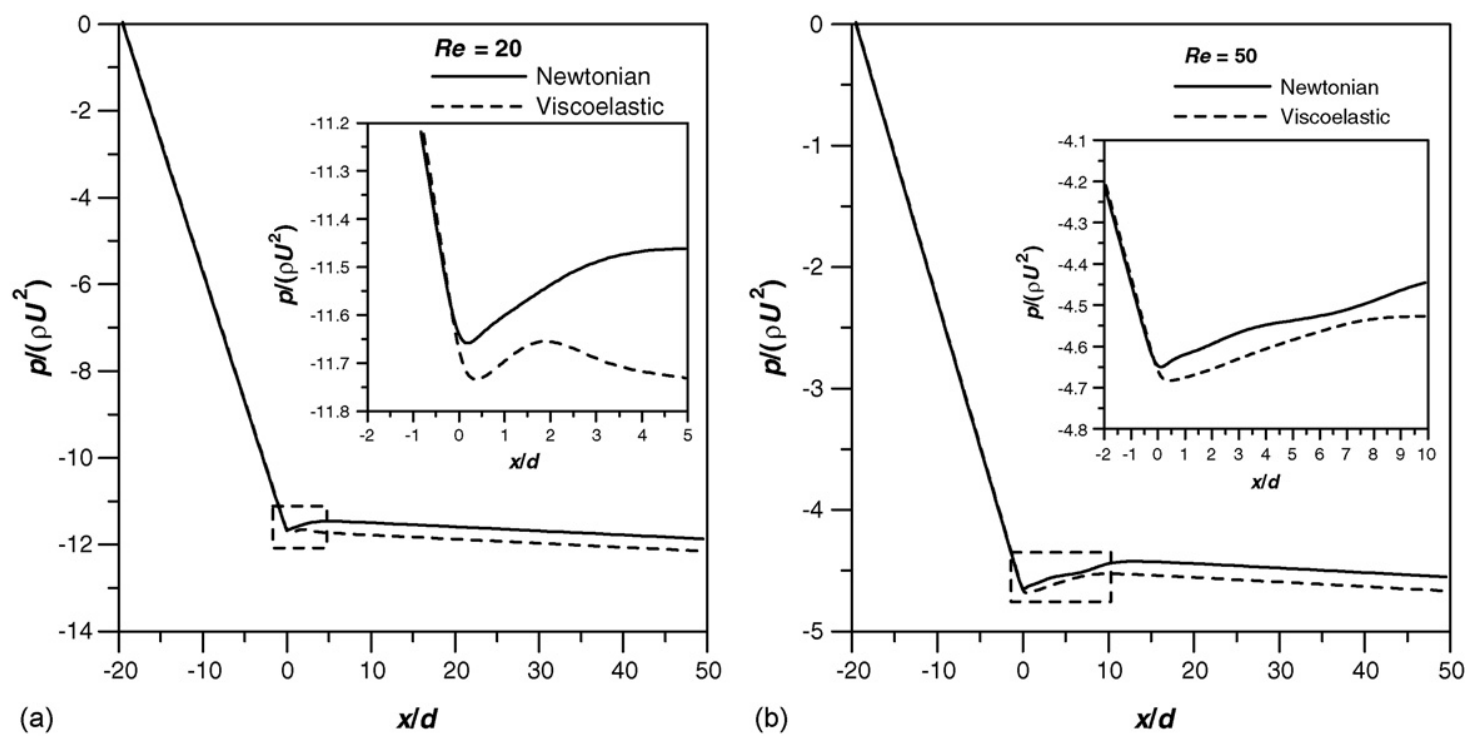

Fig. 20. Pressure distribution along the centreline for the Newtonian and viscoelastic fluids (We $=20, \beta=0.5$ and $L^{2}=100$ ), at: (a) $R e=20$ and (b) $R e=50$.

demonstrating that for the low Re numbers cases shown the bifurcation phenomenon is not sensed by the stress field. Another detail is the displacement of the maximum stress downstream of the expansion (see Fig. 17 for $W e=2$ ). The convective terms present in the stress equations tend to sweep the stresses along the flow direction even if the Reynolds number is kept constant as is the situation for the cases shown. Lastly, we can see the gradual stress concentration near to the expansion corner as the degree of elasticity increases.

In Fig. 19 we plot the variation of the normal stresses $\tau_{x x}$ and $\tau_{y y}$ along the centreline (i.e. $y=0$ ), at several Weissenberg numbers $(W e=0,1,2$ and 5) for the viscoelastic fluid $(R e=20, \beta=0.5$ and $L^{2}=100$ ). The normal stresses are non-dimensionalised by a convective scale, $\rho * U^{2}$ and for the viscoelastic cases the stresses shown correspond to the polymeric contribution only. We can see a gradual increase of the maximum transverse normal-stress $\left(\tau_{y y}>0\right)$, and the location of this maximum value moves further downstream with increasing elasticity. This behaviour is a consequence of a history (or memory) effect in the constitutive equation. The $\tau_{y y}$ contribution is dominant in the first normal-stress term (i.e. $N_{1}=\tau_{x x}-\tau_{y y}$ ) calculation and the values obtained from the stress components in Fig. 19 correspond to the elliptic region of maximum negative first normal-stress differences seen in the contours of Fig. 17, thus explaining the tendency for the lateral bulging of a fluid element seen previously (Figs. 9 and 15).

In Fig. 20 we investigate the influence of viscoelasticity and inertia on the pressure distribution along the centreline of the channel $(y=0)$ for the Newtonian and viscoelastic cases. A memory effect in the fluid can again be observed downstream of the expansion plane as a continuation of the pressure decrease for the viscoelastic liquid. Indeed, for this type of fluid we can see a lower minimum pressure, occurring slightly further downstream of the expansion compared with the Newtonian fluid, for which pressure recovery starts immediately at the expansion plane. Additionally the pressure recovery, after the expansion zone, is lower for the viscoelastic fluid, i.e. there is an enhanced pressure drop for the viscoelastic cases compared to the Newtonian case. This is an interesting result because for the corresponding contraction flow geometry, the predictions of several similar constitutive models have consistently exhibited a reduced pressure drop for viscoelastic fluids (see Alves et al. [23] for example).

\section{Conclusions}

Numerical simulations were conducted for flow in a twodimensional channel with a 1:4 sudden symmetric expansion. A symmetry-breaking bifurcation was found for both Newtonian and viscoelastic fluids, at different Reynolds numbers in each case, and represents the transition from a symmetric to an asymmetric flow. The critical Reynolds numbers at the bifurcation were $R e_{\mathrm{cr}}=36$ and 46 for the Newtonian and viscoelastic fluids $\left(L^{2}=100, W e=2\right.$ and $\left.\beta=0.5\right)$, respectively. It was shown that the critical Reynolds number decreased with increasing expansion ratio, compared with the work of Oliveira [11] for a 1:3 sudden expansion. At higher Reynolds numbers, Re higher than 64 or 73.5 for the Newtonian and viscoelastic liquid, respectively, a second bifurcation point is observed and a further recirculation regions on the "upper" wall. This effect was not observed for flows in the 1:3 expansion for $R e<100$. Comparing the Newtonian and viscoelastic fluid simulations the effect of elasticity (measured by the Weissenberg number) tends to delay the onset of the bifurcation, and the vortex length and intensity are always lower for the viscoelastic fluid when compared with the Newtonian case.

Similar conclusions can be drawn when the actual CR model is used instead of its modified version, which is mostly employed in this investigation. A comparison between these two versions for the constant viscosity FENE fluid shows almost no differences at low $R e$, up to and above the critical bifurcation point, with no systematic deviation for the CR at higher Reynolds numbers. 
It was seen that the inertial forces, through the $R e$ number, increase the length and intensity of the vortex for both Newtonian and viscoelastic fluids. In particular for the viscoelastic fluids the effect of $R e$ was inhibited in a lower range (say $R e=0-10$ ), in which the vortex size and intensity are unaffected by inertia, followed by the inertial range that parallels the Newtonian tendency for vortex enhancement. Finally, the polymer concentration was seen to have a very strong effect and for $\beta>0.8$, at $R e=40$ for example, the flow was asymmetric but for lower values of $\beta$ the asymmetry was completely removed.

\section{Acknowledgement}

The authors would like to thank the financial support by Fundação para a Ciência e a Tecnologia (FCT), Portugal, through the projects POCTI/EQU/37699/2001 and BD/22644/2005.

\section{References}

[1] D.V. Boger, K. Walters, Rheological Phenomena in Focus, Rheology Series, vol. 4, Elsevier, 1993.

[2] D. Drikakis, Bifurcation phenomena in incompressible sudden expansion flows, Phys. Fluids 9 (1997) 76-86.

[3] F. Battaglia, S.J. Tavener, A.K. Kulkarni, C.L. Merkle, Bifurcation of low Reynolds number flows in symmetric channels, AIAA J. 35 (1997) 99-105.

[4] M.S. Darwish, J.R. Whiteman, M.J. Bevis, Numerical modelling of viscoelastic liquids using a finite-volume method, J. Non-Newtonian Fluid Mech. 45 (1992) 311-337.

[5] K.A. Missirlis, D. Assimacopoulos, E. Mitsoulis, A finite volume approach in the simulation of viscoelastic expansion flows, J. Non-Newtonian Fluid Mech. 78 (1998) 91-118.

[6] P. Townsend, K. Walters, Expansion flows of non-Newtonian liquids, Chem. Eng. Sci. 49 (1994) 749-763.

[7] A. Baloch, P. Townsend, M.F. Webster, On vortex development in viscoelastic expansion and contraction flows, J. Non-Newtonian Fluid Mech. 65 (1996) 133-149.

[8] D.E. Abbott, S.J. Kline, Experimental investigation of subsonic turbulent flow over single and double backward facing steps, J. Basic Eng. 84 (1962) 317-325.

[9] W. Cherdron, F. Durst, J.H. Whitelaw, Asymmetric flows and instabilities in symmetric ducts with sudden expansions, J. Fluid Mech. 84 (1978) 13-31.

[10] F. Durst, J.C.F. Pereira, C. Tropea, The plane symmetric sudden-expansion flow at low Reynolds numbers, J. Fluid Mech. 248 (1993) 567-581.

[11] P.J. Oliveira, Asymmetric flows of viscoelastic fluids in symmetric planar expansion geometries, J. Non-Newtonian Fluid Mech. 114 (2003) 33-63.
[12] R.B. Bird, R.C. Armstrong, O. Hassager, Dynamics of Polymeric Liquids. Fluid Dynamics, vol. 1, John Wiley \& Sons, New York, 1977.

[13] R.B. Bird, C.F. Curtiss, R.C. Armstrong, O. Hassager, Dynamics of Polymeric Liquids. Kinetic Theory, vol. 2, John Wiley \& Sons, New York, 1987.

[14] M.D. Chilcott, J.M. Rallison, Creeping flow of dilute polymer solutions past cylinders and spheres, J. Non-Newtonian Fluid Mech. 29 (1988) 381-432.

[15] P.J. Coates, R.C. Armstrong, R.A. Brown, Calculation of steady-state viscoelastic flow through axisymmetric contractions with the EEME formulation, J. Non-Newtonian Fluid Mech. 42 (1992) 141-188.

[16] P.J. Oliveira, F.T. Pinho, G.A. Pinto, Numerical simulation of non-linear elastic flows with a general collocated finite-volume method, J. NonNewtonian Fluid Mech. 79 (1998) 1-43.

[17] M.A. Alves, P.J. Oliveira, F.T. Pinho, A convergent and universally bounded interpolation scheme for the treatment of advection, Int. J. Numer. Methods Fluids 41 (2003) 47-75.

[18] S.V. Patankar, D.B. Spalding, Calculation procedure for heat, mass and momentum transfer in three-dimensional parabolic flows, Int. J. Heat Mass Transfer 25 (1972) 17-87.

[19] L.F. Richardson, The approximate arithmetical solution by finite differences of physical problems involving differential equations, with an application to the stresses in a masonry dam, Trans. R. Soc. Lond. 210 (1910) 307-357.

[20] M.A. Alves, F.T. Pinho, P.J. Oliveira, Effect of a high-resolution differencing scheme on finite-volume predictions of viscoelastic flows, J. Non-Newtonian Fluid Mech. 93 (2000) 287-314.

[21] P.J. Oliveira, Method for time-dependent simulations of viscoelastic flows: vortex shedding behind cylinder, J. Non-Newtonian Fluid Mech. 101 (2001) $113-137$.

[22] J. Remmelgas, P. Singh, L.G. Leal, Computational studies of nonlinear elastic dumbbell models of Boger fluids in a cross-slot flow, J. Non-Newtonian Fluid Mech. 88 (1999) 31-61.

[23] M.A. Alves, P.J. Oliveira, F.T. Pinho, Benchmark solutions for the flow of Oldroyd-B and PTT fluids in planar contractions, J. Non-Newtonian Fluid Mech. 110 (2003) 45-75.

[24] P.S. Scott, F.A. Mirza, J. Vlachopoulos, A finite element analysis of laminar flows through planar and axisymmetric abrupt expansions, Comput. Fluids 14 (1986) 423-432.

[25] U. Cartalos, J.M. Piau, Creeping flow regimes of low concentration polymer solutions in thick solvents through an orifice die, J. Non-Newtonian Fluid Mech. 45 (1992) 231-285.

[26] P. Szabo, J.M. Rallison, E.J. Hinch, Start-up flow of a FENE-fluid through a 4:1:4 constriction in atube, J. Non-Newtonian Fluid Mech. 72 (1997) $73-86$.

[27] J.P. Rothstein, G.H. McKinley, Extensional flow of a polystyrene Boger fluid through a 4:1:4 axisymmetric contraction/expansion, J. NonNewtonian Fluid Mech. 86 (1999) 61-88

[28] M.A. Alves, R.J. Poole, Divergent flow in contractions, Under review Journal of non-Newtonian Fluid Mechanics (2006). 